\title{
Projective deformations of weakly orderable hyperbolic Coxeter orbifolds
}

\author{
SUHYOUNG CHOI \\ GYE-SEON LEE
}

\begin{abstract}
A Coxeter $n$-orbifold is an $n$-dimensional orbifold based on a polytope with silvered boundary facets. Each pair of adjacent facets meet on a ridge of some order $m$, whose neighborhood is locally modeled on $\mathbb{R}^{n}$ modulo the dihedral group of order $2 m$ generated by two reflections. For $n \geq 3$, we study the deformation space of real projective structures on a compact Coxeter $n$-orbifold $Q$ admitting a hyperbolic structure. Let $e_{+}(Q)$ be the number of ridges of order greater than or equal to 3 . A neighborhood of the hyperbolic structure in the deformation space is a cell of dimension $e_{+}(Q)-n$ if $n=3$ and $Q$ is weakly orderable, ie the faces of $Q$ can be ordered so that each face contains at most 3 edges of order 2 in faces of higher indices, or $Q$ is based on a truncation polytope.
\end{abstract}

57M50, 57N16; 53A20, 53C15

\section{Introduction}

In this paper, an $n$-orbifold $Q$ is based on a quotient space of a simply connected manifold $\widetilde{Q}$ by a discrete group $\Gamma$ acting on $\widetilde{Q}$ properly discontinuously. An orbifold structure on $Q$ is given by a covering by open sets of the form $\phi(U)$ with a model $(U, H, \phi)$, where $U$ is an open subset of $\widetilde{Q}, H$ is a finite subgroup of $\Gamma$ acting on $U$, and $\phi$ induces a homeomorphism $U / H \rightarrow \phi(U)$. Here $\widetilde{Q}$ is said to be a universal cover of $Q$, and $\Gamma$ is the fundamental group and is denoted by $\pi_{1}(Q)$.

A Coxeter group is a group that has a presentation

$$
\left\langle r_{i} \mid\left(r_{i} r_{j}\right)^{n_{i j}} \quad(i, j \in \mathbb{I})\right\rangle,
$$

where $\mathbb{I}$ is a set, $n_{i i}=1$ for each $i \in \mathbb{I}$, and $n_{i j} \in\{2,3, \ldots,+\infty\}$ is symmetric. Note that $n_{i j}=+\infty$ means there is no relation between $r_{i}$ and $r_{j}$.

A point in an $n$-orbifold $Q$ is called a silvered point if it has an open neighborhood of the form $\phi(U)$ with a model $(U, \mathbb{Z} / 2 \mathbb{Z}, \phi)$ for an open set $U$ in $\widetilde{Q}$ and a $\mathbb{Z} / 2 \mathbb{Z}-$ action on $U$ fixing a hypersurface in $U$. A Coxeter $n$-orbifold $\widehat{P}$ is an $n$-dimensional 
orbifold whose base space is an $n$-dimensional polyhedron $P$ with finitely many sides where all interior points of the facets are silvered. The fundamental group $\pi_{1}(\widehat{P})$ is isomorphic to a Coxeter group, and is generated by reflections about sides of the fundamental domain $P$. We will study only compact ones in this paper, ie closed ones. (More precisely, Davis [25; 26] calls such an orbifold a Coxeter orbifold of type III, an orbifold of reflection type or a reflectofold.)

Let $V$ be an $(n+1)$-dimensional real vector space. The projective sphere $\mathbb{S}^{n}$ is the space of rays in $V$ and is a double-cover of $\mathbb{R P}^{n}$. Let

$$
\mathrm{SL}_{n+1}^{ \pm}(\mathbb{R})=\left\{A \in \mathrm{GL}_{n+1}(\mathbb{R}) \mid \operatorname{det}(A)= \pm 1\right\} .
$$

The group $\mathrm{SL}_{n+1}^{ \pm}(\mathbb{R})$ acts on $\mathbb{S}^{n}$ effectively in the standard manner and is a doublecover of $\mathrm{PGL}_{n+1}(\mathbb{R})$. The elements of $\mathrm{SL}_{n+1}^{ \pm}(\mathbb{R})$ are the projective automorphisms of $\mathbb{S}^{n}$ and $\mathrm{SL}_{n+1}^{ \pm}(\mathbb{R})$ the projective automorphism group of $\mathbb{S}^{n}$. (We will also think of $\mathrm{SL}_{n+1}^{ \pm}(\mathbb{R})$ as a linear group when it is convenient.) Denote by $\Pi$ the natural projection from $V \backslash\{0\}$ into $\mathbb{S}^{n}$. A subspace of $\mathbb{S}^{n}$ is the image of a subspace of $V$ with the origin removed. In particular, a 2-dimensional subspace of $V$ corresponds to a great circle in $\mathbb{S}^{n}$, and an $n$-dimensional subspace gives a great $(n-1)$-sphere in $\mathbb{S}^{n}$. Furthermore, a component of the complement of a great $(n-1)$-sphere has the canonical structure of an affine $n$-space, as the complement of a codimension-one subspace of $\mathbb{R P}^{n}$ is an affine subspace. We call this an affine subspace of $\mathbb{S}^{n}$.

A convex segment in $\mathbb{S}^{n}$ is a connected arc contained in a great circle but not containing a pair of antipodal points in its interior. A subset $A$ of $\mathbb{S}^{n}$ is convex if any two points of $A$ are connected by a convex segment in $A$. An affine space has a notion of geodesics as arcs in 1-dimensional affine subspaces. A subset of an affine subspace of $\mathbb{S}^{n}$ is convex if and only if it is convex in the ordinary affine sense. A properly convex subset of $\mathbb{S}^{n}$ is a bounded convex subset of an affine subspace; see Choi [15, Chapter 2].

A side of a compact properly convex set $P$ is a maximal convex subset of the boundary of $P$. A polytope is a compact properly convex domain in $\mathbb{S}^{n}$ with finitely many sides. By a facet of a polytope, we mean a side of $P$ of codimension one. By a ridge of a polytope, we mean a side of $P$ of codimension two. (A facet will be called a face and a ridge an edge if $P$ is three-dimensional.) If $P$ is the base space of a Coxeter orbifold, then each ridge where the facets $F_{i}$ and $F_{j}$ meet will be given an order $n_{i j} \geq 2$; ie a ridge has an order $n_{i j}$ if a model neighborhood of each interior point of the ridge is given the usual product extension of the standard action of the dihedral group $D_{n_{i j}}$ of order $2 n_{i j}$ on the $2-$ plane.

Given a Lie group $G$ acting on a manifold $X$ transitively, we can consider a $(G, X)-$ structure on an orbifold $Q$ as a pair consisting of an immersion $D: \widetilde{Q} \rightarrow X$ and a 
homomorphism $h: \pi_{1}(Q) \rightarrow G$ satisfying

$$
h(\gamma) \circ D=D \circ \gamma \quad \text { for } \gamma \in \pi_{1}(Q) .
$$

For a given $(G, X)$-structure, $(D, h)$ is determined only up to the action

$$
g(D, h(\cdot))=\left(g \circ D, g h(\cdot) g^{-1}\right) \text { for } g \in G .
$$

(In each case we are considering, $D$ is an embedding.)

A real projective structure on $Q$ is a $(G, X)$-structure on $Q$ with

$$
G=\mathrm{SL}_{n+1}^{ \pm}(\mathbb{R}) \quad \text { and } \quad X=\mathbb{S}^{n}
$$

see also Section 2.1.

We can represent hyperbolic structures on an $n$-orbifold using the Klein projective model. Let the Lorentzian inner product be given by

$$
\langle x, y\rangle=-x_{1} y_{1}+x_{2} y_{2}+\cdots+x_{n+1} y_{n+1},
$$

where $x_{i}$ for $i=1, \ldots, n+1$ are components of $x \in V$ and $y_{i}$ for $i=1, \ldots, n+1$ are ones for $y \in V$. The hyperbolic space $\mathbb{H}^{n}$ is an open ball $B$ in $\mathbb{S}^{n}$ that is the image of positive time-like vectors under $\Pi$. The group of hyperbolic isometries is the subgroup $\mathrm{PO}(1, n)$ of $\mathrm{SL}_{n+1}^{ \pm}(\mathbb{R})$ acting on $B$. Hence a hyperbolic Coxeter orbifold, being of the form $\mathbb{H}^{n} / \Gamma$ for a discrete subgroup $\Gamma$ of $\mathrm{PO}(1, n)$, naturally has an induced real projective structure.

Real projective structures have been studied by many mathematicians including Kuiper [39], Benzécri [9], Koszul [38], Vinberg [47], Goldman [31], Choi and Goldman [19], Choi [13; 14] and Benoist [4]. Sometimes the topic is studied as the theory of linear representations of discrete groups, as by Koszul, Vinberg, Benoist and so on. Kac and Vinberg [36] were the first to discover hyperbolic Coxeter 2-orbifolds where the induced real projective structures deform into families of real projective structures that are not induced from hyperbolic structures. Johnson and Millson [35] constructed projective bending deformations of compact hyperbolic manifolds with embedded totally geodesic hypersurfaces. Cooper, Long and Thistlethwaite [22; 23] investigated whether the closed hyperbolic 3-manifolds of the Hodgson-Weeks census could be deformed and showed some occurrence of deformability. Benoist [6], Choi [17], Marquis [40] and Choi, Hodgson and Lee [20] investigated classes of deformable projective Coxeter orbifolds. Heusener and Porti [34] provided infinite families of hyperbolic 3-manifolds that are projectively rigid by Dehn filling; see also Ballas [1]. Surveys on real projective structures can be found in Benoist [7] and Choi [18]. 
The deformation space $\mathbb{D}(Q)$ of real projective structures on a closed orbifold $Q$ is the quotient space of the space of real projective structures on $Q$ by the action of the group of isotopies of $Q$. The space has a natural $C^{s}$-topology for $s \geq 1$; for more, see Choi [16; 18, Chapter 6].

Now we fix the dimension $n \geq 3$. Let $P$ be an $n$-dimensional complete hyperbolic convex polytope with dihedral angles that are submultiples of $\pi$; we call $P$ a hyperbolic Coxeter $n$-polytope. Then $P$ naturally has a Coxeter orbifold structure $\widehat{P}$ by silvering the facets. When a ridge has the dihedral angle $\pi / n_{i j}$, the ridge has the order $n_{i j}$. The point $t$ in $\mathbb{D}(\widehat{P})$ is hyperbolic if a hyperbolic structure on $\widehat{P}$ represents $t$.

Definition 1.1 Let $P$ be a compact hyperbolic Coxeter $n$-polytope, and let $\widehat{P}$ denote $P$ with its Coxeter orbifold structure. Suppose that $t$ is the corresponding hyperbolic point of $\mathbb{D}(\widehat{P})$. We call a neighborhood of $t$ in $\mathbb{D}(\widehat{P})$ the local deformation space of $\widehat{P}$ at $t$. We say that $\widehat{P}$ is projectively deformable at $t$, or simply deforms at $t$, if the dimension of its local deformation space at $t$ is positive. Conversely, we say that $\widehat{P}$ is locally projectively rigid at $t$, or locally rigid at $t$, if the dimension of its local deformation space at $t$ is 0 .

Definition 1.2 Let $\widehat{P}$ be a compact Coxeter 3 -orbifold with a base polytope $P$. Then $\widehat{P}$ is weakly orderable if the faces $F_{1}, \ldots, F_{f}$ of $P$ can be labeled by integers $\{1, \ldots, f\}$ so that for each face $F_{i}$, the cardinality of

$$
\mathcal{F}_{i}:=\left\{F_{j} \mid j>i \text { and the ridge } F_{i} \cap F_{j} \text { has order } 2\right\}
$$

is less than or equal to 3 .

In our case, the base polytope $P$ is always realizable as a convex polytope in an affine space since $P$ is the fundamental polytope for a properly convex real projective Coxeter orbifold. A compact properly convex $n$-polytope $P$ is called simple if exactly $n$ facets meet at each vertex. Note that compact hyperbolic Coxeter $n$-polytopes are simple. Denote by $e_{+}(\hat{P})$ the number of ridges of order greater than or equal to 3 in $\widehat{P}$.

We now state two results of the paper that follow from Theorem 4.1, the main result of the paper.

Corollary 1.1 Let $P$ be a compact hyperbolic Coxeter 3-polytope and let $\widehat{P}$ be the Coxeter orbifold arising from $P$. Suppose that $\widehat{P}$ is weakly orderable. Then a neighborhood of the hyperbolic point $t$ in $\mathbb{D}(\widehat{P})$ is a cell of dimension $e_{+}(\widehat{P})-3$.

A weakly orderable compact hyperbolic Coxeter 3 -orbifold $\widehat{P}$ is projectively deformable at $t$ if $e_{+}(\widehat{P})>3$; otherwise, it is locally rigid at $t$. 
A truncation at a vertex $v$ of a compact properly convex $n$-polytope $P$ is an operation where

- we take a hyperspace $H$ meeting only the interiors of sides of $P$ incident with $v$, and not $v$ itself,

- take the component $C$ of $P-H$ containing $v$, and

- finally delete $C$.

An iterated truncation of $P$ is an operation yielding $P_{n}$, where

$$
P=P_{0} \Rightarrow P_{1} \Rightarrow \cdots \Rightarrow P_{n}
$$

and $P_{i+1}$ is obtained from $P_{i}$ by truncation at a vertex of $P_{i}$. A truncation $n-p o l y t o p e$ is a convex $n$-polytope obtained from an $n$-simplex by iterated truncation.

Corollary 1.2 Let $P$ be a compact hyperbolic Coxeter $n$-polytope and a truncation polytope. (Assume $n \geq 3$.) Let $\widehat{P}$ be the Coxeter orbifold arising from $P$. Then a neighborhood of the hyperbolic point in $\mathbb{D}(\widehat{P})$ is a cell of dimension $e_{+}(\widehat{P})-n$.

Earlier, Marquis [40] used the word ecimahedron in place of truncation 3-polytope and showed that if $\widehat{P}$ is the Coxeter 3-orbifold arising from a compact hyperbolic Coxeter ecimahedron $P$, then $\mathbb{D}(\widehat{P})$ is diffeomorphic to $\mathbb{R}^{e_{+}(\widehat{P})-3}$. (For $n=2$, Goldman proved this result in his senior thesis.)

There is recent thesis work by Greene [32] obtaining similar results using algebrotopological methods. Also, Kapovich [37, Theorem 1] presents an analogous result for flat conformal structures.

Our main results, Corollaries 1.1 and 1.2, follow from Theorem 4.1 which will be stated and proved in Section 4.7, generalizing the notion of the weak orderability.

Almost all compact hyperbolic 3-orbifolds arising from 3-polytopes are weakly orderable. To describe this in more detail, we introduce the following terminology. An abstract 3-polyhedron is a cell complex homeomorphic to a compact 3-ball with conditions that there exists a unique 3 -cell, every 1 -cell belongs to exactly two $2-$ cells, a nonempty intersection of two 2-cells is a vertex or a 1-cell, and every 2-cell contains no fewer than three 1-cells; see Roeder, Hubbard and Dunbar [43]. It is simple if each vertex is contained in three 1-cells. The side structure of a properly convex 3-polytope $P$ gives it the structure of an abstract 3 -polytope whose $k$-cells correspond to the $k$-faces of $P$ for $k=0,1,2,3$. The boundary complex $\partial P$ of an abstract 3-polyhedron $P$ is the subcomplex of $P$ consisting of all proper cells. Let $(\partial P)^{*}$ be the dual complex of $\partial P$. A simple closed curve $\beta$ is called a $k$-circuit if it consists of $k$ edges of $(\partial P)^{*}$ for some positive integer $k$. A circuit $\beta$ is prismatic if all endpoints of the edges of $\partial P$ intersected by $\beta$ are distinct. 
Theorem 1.1 Let $P$ be a compact properly convex simple 3-polytope but not a tetrahedron. Suppose that $P$ has no prismatic 3-circuit, and has at most one prismatic 4-circuit. Let $H_{d}(P)$ be the number of compact hyperbolic Coxeter 3-orbifolds whose base polytopes are combinatorially equivalent to $P$ and the maximal edge orders are less than or equal to $d$, and let $\mathrm{WO}_{d}(P)$ denote the number of weakly orderable ones among them. Then

$$
\lim _{d \rightarrow \infty} \frac{\mathrm{WO}_{d}(P)}{H_{d}(P)}=1
$$

In particular, there exist infinitely many weakly orderable hyperbolic Coxeter 3orbifolds with base polytopes of type $P$ as above.

Question 1.1 Does the conclusion of Theorem 1.1 still hold if we assume only that $P$ is a convex simple 3-polytope?

Section 2 is a recap of some facts. In Section 2.1 we review some elementary results on orbifolds and real projective structures. In Section 2.2 we describe Vinberg's results on the general properties satisfied by real projective reflection groups. We turn Vinberg's "semialgebraic" conditions (L1) and (L2) into the "algebraic" conditions (L1) and (L2)'.

Section 3 gives various descriptions of the deformation space of real projective structures on a Coxeter orbifold $\hat{P}$. In Section 3.1 we introduce a space of representations of the fundamental group $\pi_{1}(\widehat{P})$ and identify this representation space with the deformation space of real projective structures. In Section 3.2 we introduce a solution space of some polynomial equations, a space of matrices satisfying certain conditions. We establish the equivalence of these spaces following Vinberg [47].

Section 4 discusses the results on a neighborhood of the hyperbolic structure in the deformation space of real projective structures on a compact Coxeter $n$-orbifold $\widehat{P}$. In Section 4.1 we study the Zariski tangent space of the solution space of polynomial equations giving real projective structures on $\widehat{P}$. In Section 4.2 we introduce polynomial equations defining hyperbolic structures on $\widehat{P}$, and in Section 4.3 we describe the Zariski tangent space of the solution space of these polynomial equations. In Section 4.4 we state Theorem 4.1, the main result of the paper. In Section 4.5 we compare the two Zariski tangent spaces at a hyperbolic point, and in Section 4.6 we combine this with Weil infinitesimal rigidity to prove Theorem 4.1 .

Section 5 provides several examples. In Section 5.1 we use two combinatorial results of Tutte and Fouquet-Thuillier to prove Theorem 1.1. In Section 5.2 and Section 5.3 we give examples satisfying only one of the two assumptions in Theorem 4.1. 
Acknowledgements This work has benefited from discussions and correspondences with Y Benoist, M Davis, W M Goldman, R Greene, O Guichard, S Lim, L Marquis and A Wienhard as well as many others. We especially wish to thank C D Hodgson for his advice and guidance to G-S Lee during our visit to the Department of Mathematics and Statistics at the University of Melbourne. We are also grateful to the referee for carefully reading this paper and suggesting several improvements including Question 3.1. Finally we express our gratitude to B Chriestenson for comments on our English.

S Choi was supported by the Mid-career Researcher Program through the NRF grant funded by the MEST (number R01-2008-000-10286-0). G-S Lee was supported by the National Research Foundation of Korea (NRF) grant funded by the Korean government (MEST) (number 2011-0027952), the Basic Science Research Program through the NRF grant funded by the MEST (number 2011-0004850), the Fondation Sciences Mathématiques de Paris (FSMP-IHP Program: Geometry and analysis of surface group representations) and the DFG research grant "Higher Teichmüller Theory".

\section{Preliminaries}

This section reviews the basic background material used in this article. In Section 2.1, we review some basic material on orbifolds and geometric structures; see Choi [18, Chapters 3, 4, and 6] for details. In Section 2.2 we describe Vinberg's results.

\subsection{Notation}

An orbifold $Q$ is a second countable Hausdorff space $|Q|$ with an orbifold structure, ie a covering of $|Q|$ by a collection of open sets of form $\phi(U)$ for a model $(U, H, \phi)$, where $U$ is an open subset of $\mathbb{R}^{n}$ with a finite group $H$ acting on it effectively and $\phi$ induces a homeomorphism $U / H \rightarrow \phi(U)$. We require that these models are compatible with one another in the standard way. A model $(U, H, \phi)$ is also called a chart of $Q$. A singular point is a point $x$ of $Q$ where $H$ is not trivial for every choice of a chart $(U, H, \phi)$ containing $x$.

An orbifold $Q_{1}$ covers an orbifold $Q_{2}$ by a covering map $p$ if each point of $Q_{2}$ has a connected open neighborhood $\phi_{2}\left(U_{2}\right)$ with a model $\left(U_{2}, H_{2}, \phi_{2}\right)$ such that each component $\phi_{1}\left(U_{1}\right)$ of the inverse image $U_{1}^{\prime}=p^{-1}\left(\phi_{2}\left(U_{2}\right)\right)$ has model $\left(U_{1}, H_{1}, \phi_{1}\right)$ where

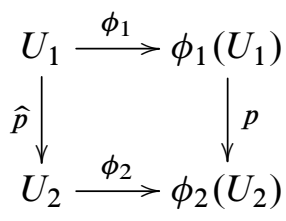


is commutative for a diffeomorphism $\hat{p}$ equivariant with respect to an injective homomorphism $\mathrm{H}_{1} \rightarrow \mathrm{H}_{2}$.

For an orbifold $Q$, we denote by $|Q|$ the base space of $Q$. Two orbifolds $Q_{1}$ and $Q_{2}$ are diffeomorphic if a homeomorphism $f:\left|Q_{1}\right| \rightarrow\left|Q_{2}\right|$ lifts to a smooth embedding for each choice of local model.

A good orbifold is an orbifold $Q$ that is covered by a manifold. It has a simply connected covering manifold $\widetilde{Q}$ called a universal cover with a covering map $p_{Q}$. The group of diffeomorphisms $f: \widetilde{Q} \rightarrow \widetilde{Q}$ so that $p_{Q} \circ f=p_{Q}$ is called the deck transformation group and is denoted by $\pi_{1}(Q)$. The base space of $Q$ is homeomorphic to the quotient space $\widetilde{Q} / \pi_{1}(Q)$.

Conversely, given a simply connected manifold $M$ and a discrete group $\Gamma$ acting on it properly discontinuously (but not necessarily freely), $M / \Gamma$ has a natural structure of an orbifold.

A geodesic in $\mathbb{R} \mathbb{P}^{n}$ is a connected arc in a 1-dimensional subspace. A geodesic in $\mathbb{S}^{n}$ is a connected arc in a 1-dimensional great circle in $\mathbb{S}^{n}$, which is a lift of a geodesic of $\mathbb{R P}^{n}$. An affine space $A^{n}$ is $\mathbb{R}^{n}$ equipped with the affine transformation group acting on it. The complement of a codimension-one subspace in $\mathbb{R P}^{n}$ can be identified with an affine space $A^{n}$. The group of projective transformations acting on $A^{n}$ is the affine transformation group. Moreover, the geodesics in $\mathbb{S}^{n}$ restricts to geodesics in $A^{n}$.

An open hemisphere in $\mathbb{S}^{n}$ is identifiable with an affine space under the double-covering map $\mathbb{S}^{n} \rightarrow \mathbb{R} \mathbb{P}^{n}$. An open hemisphere is said to be an affine subspace of $\mathbb{S}^{n}$. A polytope is a compact properly convex domain in an affine subspace with finitely many sides. (For these, the ordinary theory of convex domains in the Euclidean space applies.)

For a Lie group $G$ acting transitively on a smooth manifold $X$, a $(G, X)$-structure on an $n$-dimensional orbifold $Q$ is a maximal atlas of charts of form $(U, H, \phi)$, where $U$ is an open subset of $X$ and $H$ is a finite subgroup of $G$ acting on $U$, where

- every inclusion map $\iota: \psi(V) \rightarrow \phi(U)$ for charts $(V, J, \psi)$ and $(U, H, \phi)$ lifts to a map $k \mid V: V \rightarrow U$ for $k \in G$ equivariant with respect to a homomorphism $J \rightarrow H$, and

- each point $x \in Q$ is in $\phi(U)$, where $(U, H, \phi)$ is in the maximal atlas of the orbifold structure of $Q$ and $U \subset X$ is identified with an open set in $\mathbb{R}^{n}$ by a smooth map (a compatibility condition).

The existence of a $(G, X)$-structure implies that $Q$ is good. 
Let $\widehat{P}$ denote a compact real projective $n$-orbifold. Let $\widetilde{P}$ denote the universal cover of $\widehat{P}$ and let $\pi_{1}(\hat{P})$ denote the group of deck transformations. A real projective structure on $\widehat{P}$ gives us an immersion $D: \widetilde{P} \rightarrow \mathbb{S}^{n}$, called a developing map, and a homomorphism $h: \pi_{1}(\widehat{P}) \rightarrow \mathrm{SL}_{n+1}^{ \pm}(\mathbb{R})$, called a holonomy homomorphism, so that $D \circ \gamma=h(\gamma) \circ D$ for each $\gamma \in \pi_{1}(\widehat{P})$. Here $(D, h)$ is determined only up to the action

$$
(D, h(\cdot)) \longmapsto\left(g \circ D, g \circ h(\cdot) \circ g^{-1}\right) \quad \text { for } g \in \mathrm{SL}_{n+1}^{ \pm}(\mathbb{R}) .
$$

Conversely, the development pair $(D, h)$ determines the real projective structure; see Choi $[16 ; 17 ; 18]$ and Thurston $[44 ; 45]$ for the details.

Note the double-covering map $\mathbb{S}^{n} \rightarrow \mathbb{R} \mathbb{P}^{n}$, where the group $\mathrm{PGL}_{n+1}(\mathbb{R})$ acts on $\mathbb{R} \mathbb{P}^{n}$ transitively. We can equivalently define a real projective structure as a $(G, X)$-structure with $X=\mathbb{R} \mathbb{P}^{n}, G=\mathrm{PGL}_{n+1}(\mathbb{R})$ since $\mathrm{SL}_{n+1}^{ \pm}(\mathbb{R})$ is precisely the group consisting of automorphisms of $\mathbb{S}^{n}$ lifting elements of $\operatorname{PGL}_{n+1}(\mathbb{R})$; see Thurston [45, page 143] and Choi [13].

\subsection{Vinberg's results}

This subsection gives a summary of the groundbreaking article of Vinberg [47]; see also Benoist [8]. The English translated terminology of Vinberg is slightly different from the current one; for example the term "strictly convex" is now "properly convex." Let $V$ be an $(n+1)$-dimensional real vector space and let $V^{*}$ be its dual vector space. Let $O$ denote the origin of $V$. A cone $C$ in $V$ is a subset of $V$ with $O \in C$ so that if any point $v \in V$ is in $C$, then $s v \in C$ for each $s>0$. In our terms, the definition of a convex cone in [47] is the following. A cone $C$ in $V$ is a convex cone if $\Pi(C-\{O\})$ is a convex set in $\mathbb{S}^{n}$.

A reflection $R$ is an element of order 2 of $\mathrm{SL}_{n+1}^{ \pm}(\mathbb{R})$ which is the identity on a hyperplane of $V$. All reflections are of the form

$$
R=I_{V}-\alpha \otimes b
$$

for some linear functional $\alpha \in V^{*}$ and a vector $b \in V$ with $\alpha(b)=2$, and are in $\mathrm{SL}_{n+1}^{ \pm}(\mathbb{R})$. Observe that the kernel of $\alpha$ is the subspace $U$ of fixed points of $R$ and $b$ is the reflection vector, ie an eigenvector corresponding to the eigenvalue -1 . Hence the set of fixed points of a reflection is a subspace of codimension one in $\mathbb{S}^{n}$ and the point corresponding to the reflection vector is sent to its antipode, called the antipodal fixed point.

A rotation is an element of $\mathrm{SL}_{n+1}^{ \pm}(\mathbb{R})$ which restricts to the identity on a subspace of codimension two and acts on the complementary space by a matrix of the form $\left[\begin{array}{cc}\cos \theta & -\sin \theta \\ \sin \theta & \cos \theta\end{array}\right]$ with respect to some basis. The real number $\theta$ is the angle of the rotation. 
As a matter of notation, given a convex $n$-polytope $P$ in $\mathbb{S}^{n}$, cone $(P)$ will denote the convex polyhedral cone $\Pi^{-1}(P) \cup\{O\}$ in $V$.

Let $P$ be a properly convex $n$-polytope in $\mathbb{S}^{n}$ with sides $F_{1}, \ldots, F_{f}$ of codimension one. For each facet $F_{i}$ of $P$, take a linear functional $\alpha_{i}$ for $F_{i}$ and choose a reflection $R_{i}=I_{V}-\alpha_{i} \otimes b_{i}$ with $\alpha_{i}\left(b_{i}\right)=2$ which fixes $F_{i}$. By making a suitable choice of signs, we may assume that $P$ is defined by the inequalities

$$
\alpha_{i} \geq 0, \quad i \in \mathbb{I}=\{1, \ldots, f\} .
$$

The group $\Gamma \subset \mathrm{SL}_{n+1}^{ \pm}(\mathbb{R})$ generated by all these reflections $R_{i}$ is called a (real) projective Coxeter group if

$$
\gamma \stackrel{\circ}{P} \cap \stackrel{\circ}{P}=\varnothing \quad \text { for every } \gamma \in \Gamma \backslash\{1\},
$$

where $\stackrel{\circ}{P}$ is the interior of $P$. Note that Vinberg [47] used the term linear Coxeter group in place of projective Coxeter group. The $f \times f$ matrix $A=\left(a_{i j}\right), a_{i j}=\alpha_{i}\left(b_{j}\right)$, is called the Cartan matrix of the group $\Gamma$ and $P$ is called a fundamental chamber of $\Gamma$. For $x \in P$, let $\Gamma_{x}$ denote the subgroup of $\Gamma$ generated by $\left\{R_{i} \mid x \in F_{i}\right\}$. Define $P^{s}=\left\{x \in P \mid \Gamma_{x}\right.$ is finite $\}$.

Theorem 2.1 [47, Theorem 1 and Propositions 6 and 17] The following conditions are necessary and sufficient for any group $\Gamma$, generated by the reflections $R_{1}, \ldots, R_{f}$ fixing respectively facets $F_{1}, \ldots, F_{f}$ of the properly convex $n$-polytope $P$, to be a projective Coxeter group:

(L1) $a_{i j} \leq 0$ for $i \neq j$, and $a_{i j}=0$ if and only if $a_{j i}=0$.

(L2) $a_{i i}=2$, and for $i, j$ with $i \neq j$,

(i) if $F_{i}$ and $F_{j}$ are adjacent, ie meet in a ridge, then $a_{i j} a_{j i}=4 \cos ^{2}\left(\frac{\pi}{n_{i j}}\right)<4$ for an integer $n_{i j} \geq 2$,

(ii) else $a_{i j} a_{j i} \geq 4$.

Proof [47, Proposition 17] gives the necessity of the conditions (L1) and (L2).

Given (L1) and (L2), [47, Proposition 7 and Theorem 1] show that $\Gamma$ is a projective Coxeter group with the fundamental chamber $P$; see also [8, Theorem 1.5].

In fact, if $a_{i j} a_{j i}=4 \cos ^{2}\left(\pi / n_{i j}\right)$, then the product $R_{i} R_{j}$ is a rotation of angle $2 \pi / n_{i j}$ and the group generated by the two reflections $R_{i}$ and $R_{j}$ is the dihedral group $D_{n_{i j}}$. In particular, if $a_{i j}=a_{j i}=0$ then $R_{i} R_{j}$ is a rotation of angle $\pi / 2$ and $R_{i}$ and $R_{j}$ generate a dihedral group of order 4 , ie a Klein four group. If $a_{i j} a_{j i} \geq 4$ then $R_{i}$ and $R_{j}$ generate an infinite group and $n_{i j}=+\infty$; see [47, Section 2]. 
The group generated by $R_{1}, \ldots, R_{f}$ is isomorphic to a Coxeter group, and is also called the projective reflection group generated by $R_{1}, \ldots, R_{f}$.

For each reflection $R_{i}, \alpha_{i}$ and $b_{i}$ are determined up to a positive scalar by

$$
\alpha_{i} \mapsto d_{i} \alpha_{i}, \quad b_{i} \mapsto d_{i}^{-1} b_{i} \quad \text { with } d_{i}>0 .
$$

Hence the Cartan matrix $A$ of $\Gamma$ is determined up to the conjugation action of a group of diagonal $f \times f$ matrices with positive diagonal entries.

Theorem 2.2 [47, Theorem 2] Let $\Gamma$ be a projective Coxeter group and $P$ its fundamental chamber. The subset $\left\{x \in P \mid \Gamma_{x}\right.$ is finite $\}$ is denoted by $P^{s}$. Then the following statements hold:

- $\Omega_{\Gamma}=\bigcup_{\gamma \in \Gamma} \gamma P$ is convex.

- $\quad \Gamma$ is a discrete subgroup of $\mathrm{SL}_{n+1}^{ \pm}(\mathbb{R})$ preserving the interior $\stackrel{\circ}{\Omega}_{\Gamma}$ of $\Omega_{\Gamma}$.

- $\stackrel{\circ}{\Omega}_{\Gamma} \cap P=P^{s}$, and is homeomorphic to $\stackrel{\circ}{\Omega}_{\Gamma} / \Gamma$.

An easy consequence of the theorem is that the group $\Gamma$ acts on $\AA_{\Gamma}$ properly discontinuously. Thus $\AA_{\Gamma}$ gives a convex open subset of the projective sphere $\mathbb{S}^{n}$, and $\stackrel{\circ}{\Omega}_{\Gamma} / \Gamma$ determines a convex real projective structure on the Coxeter $n$-orbifold with the fundamental domain homeomorphic to $P^{s}$. For example, let $P$ be a hyperbolic Coxeter $n$-polytope of finite volume. Suppose that $\Gamma$ is the discrete group generated by the isometric reflections with respect to facets of $P$ in the hyperbolic space $\mathbb{H}^{n}$ in the Klein model in $\mathbb{S}^{n}$. Then $\stackrel{\circ}{\Omega}_{\Gamma}=\mathbb{H}^{n}$ and $\stackrel{\circ}{\Omega}_{\Gamma} / \Gamma$ is a hyperbolic Coxeter $n$-orbifold.

A projective Coxeter group $\Gamma$ is elliptic, parabolic and hyperbolic if $\Gamma$ is conjugate to a discrete group generated by reflections in the sphere, the Euclidean space and the hyperbolic space respectively, provided that neither any proper plane in the hyperbolic space nor any point at infinity is $\Gamma$-invariant.

A Cartan matrix is indecomposable if it is not a direct sum of two matrices. Thus every matrix $A$ decomposes into a direct sum of indecomposable matrices, which are components of $A$. By Frobenius's theorem, any indecomposable matrix $A$ satisfying condition (L1) has a real eigenvalue (see Gantmacher [29]). An indecomposable Cartan matrix $A$ is positive, zero and negative type if the smallest real eigenvalue is positive, zero and negative respectively. Denote by $A^{+}$(resp. $A^{0}, A^{-}$) the direct sum of its components of positive type (resp. zero type, negative type). Any matrix $A$ satisfying condition (L1) is a direct sum of $A^{+}, A^{0}$ and $A^{-}$. 
Proposition 2.1 [47, Proposition 22] Let $\Gamma$ be a projective Coxeter group with a properly convex $n$-dimensional fundamental chamber in $\mathbb{S}^{n}$, and let $A$ be the Cartan matrix of $\Gamma$. Then $\Gamma$ is elliptic if and only if $A=A^{+}$if and only if $\Gamma$ is finite.

Proposition 2.2 [47, Proposition 23] Let $\Gamma$ be a projective Coxeter group with a properly convex $n$-dimensional fundamental chamber in $\mathbb{S}^{n}$, and let $A$ be the Cartan matrix of $\Gamma$. Then $\Gamma$ is parabolic if and only if $A=A^{0}$ and $\operatorname{rank} A=n$.

We shall consider only the case when $P=P^{s}$, or equivalently, $\Omega_{\Gamma}=\stackrel{\circ}{\Omega}_{\Gamma}$; we call $\Gamma$ perfect. The following three statements are equivalent.

(1) $\Gamma$ is perfect.

(2) The base space $P^{S}$ of the associated orbifold $\widehat{P}$ equals $P$ exactly.

(3) $\widehat{P}$ is compact.

The following is a fairly well-known and commonly used consequence of [47].

Proposition 2.3 [47, Lemma 15 and Propositions 19 and 26] Let $\Gamma$ be a perfect projective Coxeter group with a properly convex $n$-dimensional fundamental chamber $P$ in $\mathbb{S}^{n}$ and let $A$ be the Cartan matrix of $\Gamma$. Then exactly one of the following statements holds.

- $\Gamma$ is elliptic.

- $\Gamma$ is parabolic.

- $A$ is indecomposable and of negative type, and $\operatorname{rank} A=\operatorname{dim} V=n+1$.

Moreover, if $\Gamma$ is neither elliptic nor parabolic, then $\Gamma$ is irreducible and $\Omega_{\Gamma}$ is properly convex.

Proof By [47, Proposition 26], we have only the above three possibilities or $\Gamma$ is the direct product of a parabolic group and $\mathbb{Z} / 2 \mathbb{Z}$. In this case, $\Gamma$ is not perfect as we can see from [47, Lemma 17].

In the third case, we only have to prove the last statement. Since our fundamental domain $P$ is properly convex, [47, Proposition 18] implies that $\Gamma$ is reduced. The last statement follows from [47, Lemma 15].

Let $P$ be a properly convex $n$-polytope in $\mathbb{S}^{n}$ and the polyhedral cone $K=\operatorname{cone}(P)$ be given. Again a side of $K$ is a maximal convex subset of $K$. The complex of $K$, 
denoted by $\mathfrak{F} K$, is the set of its (closed) sides, partially ordered by inclusion. Let $K_{1}, \ldots, K_{f}$ be the facets of $K$, and let $\mathbb{I}=\{1, \ldots, f\}$. For any sides $L$ of $K$, define

$$
\sigma(L)=\left\{i \in \mathbb{I} \mid K_{i} \supset L\right\} \quad \text { and } \quad \sigma(\mathfrak{F} K)=\{\sigma(L) \subset \mathbb{I} \mid L \in \mathfrak{F} K\} .
$$

For any subset $S$ of $\mathbb{I}$, the standard subgroup $\Gamma_{S}$ of $\Gamma$ is the subgroup generated by the reflection $R_{i}, i \in S$, and the principal submatrix $A_{S}$ of $A$ is the submatrix of $A$ consisting of the entries $a_{i j}$ for each $i, j \in S$. Denote by $S^{+}\left(\right.$resp. $S^{0}, S^{-}$) the subset $T$ of $S$ such that $A_{T}=A_{S}^{+}$(resp. $A_{S}^{0}, A_{S}^{-}$). We define $Z(S):=\{i \in \mathbb{I} \mid$ $a_{i j}=0$ for each $\left.j \in S\right\}$.

Proposition 2.4 [47, Theorems 4] Let $\Gamma$ be a projective Coxeter group, let $P$ be its fundamental chamber and let $K$ be cone $(P)$. Assume that a subset $S$ of $\mathbb{I}$ satisfies two conditions: $S=S^{0}$ and $Z(S)^{0}=\varnothing$. Then $S \in \sigma(\mathfrak{F} K)$.

Proposition 2.5 [47] Let $\Gamma$ be a perfect projective Coxeter group, let $P$ be its fundamental chamber and let $K=\operatorname{cone}(P)$. Then $S \in \sigma(\mathfrak{F} K)$ if and only if $\Gamma_{S}$ is finite or $S=\mathbb{I}$.

Proof This is the statement of [47, Equation 8].

Lemma 2.1 Let $\Gamma$ be a perfect projective Coxeter group, and let $A$ be the Cartan matrix of $\Gamma$. If $A$ has a principal submatrix of zero type, then $\Gamma$ is parabolic.

Proof Suppose that $S=S^{0}$ for some nonempty $S \subset \mathbb{I}$. Define $T:=\mathrm{Z}(S)^{0}$. Observe that $S \cup T=(S \cup T)^{0}$ and $\mathrm{Z}(S \cup T)^{0}=\varnothing$, and thus by Proposition 2.4, $S \cup T \in \sigma(\mathfrak{F} K)$ with $K=\operatorname{cone}(P)$.

Suppose that $S \cup T \neq \mathbb{I}$. Then $\Gamma_{S}$ is finite by Proposition 2.5, and $S=S^{0}$ should be empty, a contradiction.

If we have $S \cup T=\mathbb{I}$, then $\Gamma$ is either elliptic or parabolic by Proposition 2.3 as $\mathbb{I}^{0}=\mathbb{I}$. If $\Gamma$ is elliptic, then $\mathbb{I}^{+}=\mathbb{I}$, a contradiction as $S$ is not empty. Hence $\Gamma$ is parabolic; see also the proof of [47, Theorem 7].

Proposition 2.6 Let $\Gamma$ be a perfect projective Coxeter group, and let $A=\left(a_{i j}\right)$ be the Cartan matrix of $\Gamma$. If $\Gamma$ is not parabolic, then $a_{i j} a_{j i}>4$ holds if $F_{i}$ and $F_{j}$ are not adjacent and $i \neq j$.

Proof If $a_{i j} a_{j i}=4$ holds for some $i \neq j$, then the principal submatrix $\left[\begin{array}{cc}2 & a_{i j} \\ a_{j i} & 2\end{array}\right]$ of $A$ is of zero type. By Lemma 2.1, $\Gamma$ is parabolic. 
Proposition 2.6 shows that for negative-type perfect projective Coxeter groups, we can now replace the semialgebraic condition (L2) with an open condition (L2) ${ }^{\prime}$ where we replace (L2)(ii) with:

(L2)' (ii) If $F_{i}$ and $F_{j}$ are not adjacent, then $a_{i j} a_{j i}>4$.

The following was one of the main results of [47].

Theorem 2.3 [47, Corollary 1] Let $A$ be an $f \times f$ matrix satisfying (L1) and (L2), and let $\operatorname{rank} A=n+1$. Suppose that $A$ has no component of zero type. Then there exists a projective Coxeter group $\Gamma \subset \mathrm{SL}_{n+1}^{ \pm}(\mathbb{R})$ with the Cartan matrix $A$. Furthermore, $\Gamma$ is unique up to the conjugations in $\mathrm{SL}_{n+1}^{ \pm}(\mathbb{R})$.

\section{Deformation spaces of real projective structures}

Through this section, we give three descriptions of the deformation space of real projective structures on a compact $n$-dimensional Coxeter orbifold $\widehat{P}$, when $\widehat{P}$ admits a real projective structure but does not admit a spherical or Euclidean structure. In Section 3.1, we describe the deformation space in terms of representations from $\pi_{1}(\widehat{P})$ into $\mathrm{SL}_{n+1}^{ \pm}(\mathbb{R})$. In Section 3.2, we describe this representation space in terms of polynomial equations and Cartan matrices following Vinberg respectively.

\subsection{Deformation spaces and the representation spaces}

We restate the results of Vinberg [47] for perfect groups under the orbifold viewpoint.

Proposition 3.1 (Vinberg) Let $\widehat{P}$ be a compact real projective Coxeter $n$-orbifold where $\hat{P}$ does not admit a spherical or Euclidean structure. Then each developing map $D$ of the universal cover $\widetilde{P}$ of $\widehat{P}$ is a diffeomorphism to an open properly convex domain in $\mathbb{S}^{n}$. Furthermore, $D(P)$ is a fundamental chamber for the fundamental domain $P$ of $\widetilde{P}$, a properly convex $n$-polytope, the projective Coxeter group $h\left(\pi_{1}(\hat{P})\right)$, where $h: \pi_{1}(\widehat{P}) \rightarrow \mathrm{SL}_{n+1}^{ \pm}(\mathbb{R})$ is the holonomy homomorphism associated with $D$.

Given a Coxeter orbifold $\widehat{P}$, the choice of the fundamental polytope $P$ in the universal cover gives us the fundamental set of generators in $\pi_{1}(\widehat{P})$ associated with each facet of $P$. They are labeled by $r_{1}, \ldots, r_{f}$, where $f$ is the number of facets of $P$. Call these the fundamental generators. Since we can imbed $\operatorname{Hom}\left(\pi_{1}(\widehat{P}), \mathrm{SL}_{n+1}^{ \pm}(\mathbb{R})\right)$ as an algebraic subset of $\mathrm{SL}_{n+1}^{ \pm}(\mathbb{R})^{f}$ for the number $f$ of fundamental generators of 
$\pi_{1}(\widehat{P})$, we let $\operatorname{Hom}\left(\pi_{1}(\widehat{P}), \mathrm{SL}_{n+1}^{ \pm}(\mathbb{R})\right)$ be a real algebraic set with the standard pointset topology of the subspace.

The $\mathrm{SL}_{n+1}^{ \pm}(\mathbb{R})$-action on $\operatorname{Hom}\left(\pi_{1}(\hat{P}), \mathrm{SL}_{n+1}^{ \pm}(\mathbb{R})\right)$ by conjugation is not effective since $\pm I_{V}$ is in the kernel and

$$
\operatorname{Hom}\left(\pi_{1}(\widehat{P}), \mathrm{SL}_{n+1}^{ \pm}(\mathbb{R})\right) / \mathrm{SL}_{n+1}^{ \pm}(\mathbb{R})
$$

is equivalent to

$$
\operatorname{Hom}\left(\pi_{1}(\widehat{P}), \mathrm{SL}_{n+1}^{ \pm}(\mathbb{R})\right) / \mathrm{PGL}_{n+1}(\mathbb{R}) .
$$

We will study the later space only.

A discrete subgroup $\Gamma$ of $\mathrm{SL}_{n+1}^{ \pm}(\mathbb{R})$ is dividing if $\Gamma$ acts faithfully and properly discontinuously on a properly convex open subset $\Omega$ of $\mathbb{S}^{n}$ so that the quotient $\Omega / \Gamma$ is compact; see Benoist [4]. Let $D_{\text {rep }}^{\prime}(\widehat{P})$ denote the space of dividing faithful representations of $\pi_{1}(\widehat{P})$. Define the subspace $D_{\text {rep }}(\widehat{P})$ by $h \in D_{\text {rep }}(\widehat{P})$ if $h$ is discrete and faithful and $h\left(\pi_{1}(\widehat{P})\right)$ acts as a dividing projective Coxeter group on a properly convex domain.

Question 3.1 Is $D_{\text {rep }}^{\prime}(\widehat{P})=D_{\text {rep }}(\widehat{P})$ for a compact Coxeter $n$-orbifold $\widehat{P}$ ?

We combine the works of Benoist, Charney and Davis, Choi, Koszul, Qi and finally Davis to prove the following theorem. Let $\mathbb{D}(\hat{P})$ be the deformation space of real projective structures on $\widehat{P}$. (Of course, this set could be empty.)

Theorem 3.1 Let $\widehat{P}$ be a compact Coxeter $n$-orbifold. Assume that $\widehat{P}$ admits a real projective structure, but does not admit a spherical or Euclidean structure.

- $D_{\text {rep }}(\widehat{P})$ is a union of components of $\operatorname{Hom}\left(\pi_{1}(\widehat{P}), \mathrm{SL}_{n+1}^{ \pm}(\mathbb{R})\right), \mathrm{PGL}_{n+1}(\mathbb{R})$ acts properly and freely on it, and the quotient space $D_{\text {rep }} / \mathrm{PGL}_{n+1}(\mathbb{R})$ is a Hausdorff space.

- $\mathbb{D}(\widehat{P}) \rightarrow D_{\text {rep }}(\widehat{P}) / \mathrm{PGL}_{n+1}(\mathbb{R})$ is a homeomorphism.

- For each element $h: \pi_{1}(\widehat{P}) \rightarrow \mathrm{SL}_{n+1}^{ \pm}(\mathbb{R})$ of $D_{\text {rep }}(\widehat{P})$, the sphere $\mathbb{S}^{n}$ contains a unique properly convex open subset $\Omega$ of $\mathbb{S}^{n}$ so that $\Omega / h\left(\pi_{1}(\widehat{P})\right)$ is diffeomorphic to $\hat{P}$. Here $\Omega$ is determined up to the antipodal map $\mathcal{A}:=-I_{V}$

Proof The fundamental group $\pi_{1}(\widehat{P})$ of $\widehat{P}$ is an infinite, nonaffine and irreducible Coxeter group by Proposition 2.3. Hence, by Qi [41, Theorem 1.1], the center of any finite-index subgroup of $\pi_{1}(\hat{P})$ is trivial, and so by Benoist [4, Theorem 2.2; 5, Theorem 1.1], $D_{\text {rep }}^{\prime}(\widehat{P})$ is a union of components of $\operatorname{Hom}\left(\pi_{1}(\widehat{P}), \mathrm{SL}_{n+1}^{ \pm}(\mathbb{R})\right)$, consisting of dividing discrete faithful representations. (For each element $h \in D_{\text {rep }}^{\prime}(\widehat{P}), \Omega / h\left(\pi_{1}(\widehat{P})\right.$ ) is a compact orbifold when $\Omega$ is a properly convex domain by Benoist [5].) 
Now, $D_{\text {rep }}(\widehat{P})$ is an open subset of $D_{\text {rep }}^{\prime}(\widehat{P})$ by Koszul [38]; see Choi [17]. The subset is closed in the second space: let $h_{i}$ be a sequence of representations in $D_{\text {rep }}(\widehat{P})$ converging to an element $h$ of $D_{\text {rep }}^{\prime}(\hat{P})$. For a set of fundamental generators $r_{j}$, $j=1, \ldots, f, h_{i}\left(r_{j}\right)$ is a reflection fixing points of a side $F_{j, i}$ of a compact convex polytope $P_{i}$. Let $\Omega_{i}$ be a properly convex domain in $\mathbb{S}^{n}$ where $h_{i}\left(\pi_{1}(\widehat{P})\right)$ acts as a projective Coxeter group. We have $h_{i}\left(r_{j}\right) \rightarrow h\left(r_{j}\right)$ for each $j$, where $h\left(r_{j}\right)$ is a reflection and fixes points of a hyperspace $H_{j}$. Since $h\left(\pi_{1}(\widehat{P})\right), h \in D_{\text {rep }}^{\prime}(\widehat{P})$, acts on a properly convex open domain $\Omega \subset \mathbb{S}^{n}$, each $H_{j}$ meets $\Omega$. Here $\left\{H_{l}\right\}_{l=1, \ldots, f}$ are mutually distinct since otherwise we loose the faithfulness of the action.

Denote by $H_{l, i}$ the hyperspace in $\mathbb{S}^{n}$ fixed by $h_{i}\left(r_{l}\right)$. For a subset $S$ of $\{1, \ldots, f\}$, let $\Gamma_{S}$ denote the subgroup of $\pi_{1}(\widehat{P})$ generated by $r_{l}$ for $l \in S$. Since $h_{i}\left(\pi_{1}(\widehat{P})\right)$ acts properly discontinuously on $\Omega, h_{i}\left(\Gamma_{S}\right)$ is finite if $\bigcap_{l \in S} H_{l, i} \cap \Omega_{i} \neq \varnothing$. The converse is true by Vinberg [47, Theorem 7], and the condition also implies $\bigcap_{l \in S} F_{l, i} \neq \varnothing$. Thus, the combinatorial intersection pattern of $\left\{H_{l, i} \cap \Omega_{i}\right\}_{l=1, \ldots, f}$ is the same as that of facets $\left\{F_{l, i}\right\}_{l=1, \ldots, f}$ for $P_{i}$; see also Davis [24, Example 7.1.4]. Similarly, $h\left(\Gamma_{S}\right)$ is finite if $\bigcap_{l \in S} H_{l} \cap \Omega \neq \varnothing$. The converse is also true: we consider the properly convex cone $\Pi^{-1}(\Omega) \cup\{O\}$. Given a linear finite group action on $\Pi^{-1}(\Omega) \cup\{O\}$ with the subspace $P$ of fixed points, $\operatorname{dim} P \geq 1$, we must have $P \cap \Pi^{-1}(\Omega) \neq \varnothing$. Hence, $\bigcap_{l \in S} H_{l} \cap \Omega_{i} \neq \varnothing$. We thus have

$$
\bigcap_{l \in J} F_{l, i} \neq \varnothing \Leftrightarrow \bigcap_{l \in J} H_{l, i} \cap \Omega_{i} \neq \varnothing \Leftrightarrow \bigcap_{l \in J} H_{l} \cap \Omega \neq \varnothing
$$

for any subset $J$ of $\{1, \ldots, f\}$.

We may also assume that the sequence $\left\{\bar{\Omega}_{i}\right\}$ of the closures of $\Omega_{i}$ geometrically converges to a compact convex set $K$ by choosing a subsequence; see Choi [15, Propositions 2.8 and 2.10]. The set $K$ is properly convex and has nonempty interior since otherwise $h$ is reducible; see Choi and Goldman [19, Lemma 1] and what follows. We may identify $K=\bar{\Omega}$. Also, we assume that $\left\{P_{i}\right\}$ geometrically converges to a compact convex set $P^{\prime}$ in $\mathbb{S}^{n}$. Hence, $P^{\prime}$ is properly convex since $P^{\prime} \subset \bar{\Omega}$. By taking a subsequence if necessary, we may assume that each sequence $\left\{S_{i}\right\}$ of sides of $P_{i}$ geometrically converges to a compact convex subset $S_{\infty}$ of $\mathbb{S}^{n}$. If $\operatorname{dim} S_{i}=0$ for all $i$, then $S_{\infty} \in \Omega$ by (3-1). Since $S_{\infty}$ is a properly convex set, we can deduce that $S_{\infty}$ is the convex hull of its vertices. Since $\Omega$ is properly convex also, we obtain $S_{\infty} \subset \Omega$. Any sequence $\left\{\left(S_{i}, T_{i}\right)\right\}$ of disjoint pairs of sides of $P_{i}$ geometrically converges to a disjoint pair of subsets by (3-1). We deduce that $\bigcap_{l \in J} F_{l, 1} \neq \varnothing$ if and only if $\bigcap_{l \in J} F_{l, \infty} \neq \varnothing$. The facets of $P^{\prime}$ have the same intersection pattern as $\left\{H_{l, 1} \cap \Omega_{1}\right\}_{l=1, \ldots, f}$. Also, $P^{\prime} \cap \Omega=P^{\prime}$ since otherwise $\Omega / h\left(\pi_{1}(\widehat{P})\right)$ is not compact. Hence $h\left(\pi_{1}(\widehat{P})\right)$ is a projective Coxeter group based on $P^{\prime}$. 
By Lemma 3.1, the conjugation action by $\operatorname{PGL}_{n+1}(\mathbb{R})$ is proper and free. This proves the first item.

The holonomy homomorphism $h$ is in $D_{\text {rep }}(\widehat{P})$ by Proposition 3.1. By Choi [16, Theorem 1] and the first item, the map from a real projective structure to its holonomy homomorphism induces a local homeomorphism

$$
\text { hol: } \mathbb{D}(\widehat{P}) \rightarrow D_{\text {rep }}(\widehat{P}) / \operatorname{PGL}_{n+1}(\mathbb{R}) .
$$

Now we show that hol is injective. Suppose that $\Omega_{k}$ for each $k=1,2$ is a properly convex open subset of $\mathbb{S}^{n}$ on which $\Gamma:=h\left(\pi_{1}(\widehat{P})\right)$ acts for $h \in D_{\text {rep }}(\widehat{P})$ as a dividing projective Coxeter group. Let $\widetilde{\Gamma}$ denote the torsion-free finite index subgroup by Selberg's lemma.

If $\Omega_{1} \cap \Omega_{2} \neq \varnothing$, then $\Omega^{\prime}=\Omega_{1} \cap \Omega_{2}$ is a connected properly convex open domain where $\Gamma$ acts properly discontinuously. Each map $\Omega^{\prime} / \widetilde{\Gamma} \rightarrow \Omega_{k} / \widetilde{\Gamma}, k=1,2$ of closed manifolds is surjective by a homology theory since both are $K(\widetilde{\Gamma}, 1)$-spaces. This implies that $\Omega_{1}=\Omega_{2}$ or $\Omega_{1} \cap \Omega_{2}=\varnothing$; see the proof of Cooper and Delp [21, Proposition 2.2]. Since the antipodal map $\mathcal{A}: \mathbb{S}^{n} \rightarrow \mathbb{S}^{n}$ conjugates from $h\left(\pi_{1}(\widehat{P})\right)$ to itself, $\Omega_{2}=\mathcal{A}\left(\Omega_{1}\right)$ or $\Omega_{2} \cap \mathcal{A}\left(\Omega_{1}\right)=\varnothing$ by the same reasoning.

Assume that $\Omega_{1} \cap \Omega_{2}=\varnothing$ and $\Omega_{2} \cap \mathcal{A}\left(\Omega_{1}\right)=\varnothing$. By Benoist [3, Proposition 1.1], $\Gamma$ contains an element $\gamma$ with an attracting fixed point $y$ in the boundary of $\Omega_{1}$ so that the eigenvalue of the vector in the direction of $y$ has a norm strictly greater than those of all other eigenvalues. The element $\gamma$ acts on a great $(n-1)$-sphere $S$ whose complement contains $y$. The pair $y$ and its antipode $y_{-}$are the unique attracting fixed points of the components of $\mathbb{S}^{n}-S$ containing them respectively. We can choose a point $z$ in $\Omega_{2}-S$. As $m \rightarrow \infty$, the sequence $\gamma^{m}(z)$ converges to $y$ or $y_{-}$. Thus, $y \in \bar{\Omega}_{2} \cap \bar{\Omega}_{1} \neq \varnothing$ or $y_{-} \in \bar{\Omega}_{2} \cap \mathcal{A}\left(\bar{\Omega}_{1}\right) \neq \varnothing$. The nonempty set gives a $\Gamma$-invariant convex subset of dimension less than $n$; however, $h$ is irreducible by Proposition 2.3. This is a contradiction.

Therefore, $\Omega_{2}=\Omega_{1}$ or $\Omega_{2}=\mathcal{A}\left(\Omega_{1}\right)$. Hence, $\Omega_{2} / \Gamma=\Omega_{1} / \Gamma$ or $\mathcal{A}$ induces a projective diffeomorphism $\Omega_{2} / \Gamma \rightarrow \Omega_{1} / \Gamma$. This proves the injectivity of hol.

The surjectivity of hol is shown as follows. By definition, each element of $D_{\text {rep }}(\widehat{P})$ acts cocompactly on a properly convex open subset of $\mathbb{S}^{n}$ as a projective Coxeter group. We now show that the quotient orbifold is diffeomorphic to $\widehat{P}$. Consider $\widehat{P}_{h}:=\Omega / h\left(\pi_{1}(\widehat{P})\right)$ for $h$ in $D_{\text {rep }}(\widehat{P})$. Since $h\left(\pi_{1}(\widehat{P})\right)$ is isomorphic to $\pi_{1}(\widehat{P})$, by Charney and Davis [12], the Coxeter diagrams are the same for the two groups, and a properly convex fundamental domain $F_{h}$ of $\hat{P}_{h}$ has the same facial incident relation as that of a properly convex fundamental domain $F$ of $\widehat{P}$; see Davis [24, Section 13.1]. 
By Davis [26, Corollary 1.3], $\widehat{P}_{h}$ is diffeomorphic to $\widehat{P}$; see also Wiemeler [51, Corollary 5.3]. Therefore, hol is a homeomorphism as hol is a local homeomorphism.

The third item was proved while proving the second one.

The following lemma is a generalization of Choi [17, Lemma 1].

Let $R(n+1)$ denote the subspace $\mathrm{SL}_{n+1}^{ \pm}(\mathbb{R})$ of all reflections. For each element $g \in \mathrm{SL}_{n+1}^{ \pm}(\mathbb{R})$, let $[g] \in \mathrm{PGL}_{n+1}(\mathbb{R})$ denote the corresponding element.

Lemma 3.1 Let $\mathcal{U} \subset R(1+n)^{f}$ denote the subspace of all $\left(g_{1}, \ldots, g_{f}\right)$ generating an irreducible dividing projective Coxeter group $\Gamma$. Then the $\operatorname{PGL}_{n+1}(\mathbb{R})$-action on $\mathcal{U}$ by conjugation

$$
[g] \circ\left(g_{1}, \ldots, g_{f}\right)=\left(g g_{1} g^{-1}, \ldots, g g_{f} g^{-1}\right), \quad g \in \mathrm{SL}_{n+1}^{ \pm}(\mathbb{R})
$$

is proper and free.

Proof The proof for the properness directly generalizes that of Choi [17, Lemma 1] as the group $\Gamma$ is irreducible.

Suppose that an element $\widetilde{g}$ of $\mathrm{SL}_{n+1}^{ \pm}(\mathbb{R})$ satisfies $\widetilde{g} g_{i}=g_{i} \widetilde{g}$ for $i=1, \ldots, f$. We have a compact properly convex polytope $P$ as a properly convex fundamental domain of $\Gamma$ since $\Gamma$ is a dividing projective Coxeter group. Choosing generators differently if necessary, we may assume without loss of generality that each side $S_{i}$ of $P$ is fixed by $g_{i}$ for $i=1, \ldots, f$. Since $\tilde{g}$ commutes with $g_{i}, \widetilde{g}$ acts on the subspace $S_{i}^{\prime} \subset \mathbb{S}^{n}$ containing $S_{i}$ and each pair $\left\{r_{i}, \mathcal{A}\left(r_{i}\right)\right\}$ of antipodal fixed points of $g_{i}$. Therefore, $\tilde{g}$ acts on $\left\{v_{1}, \ldots, v_{m}, \mathcal{A}\left(v_{1}\right), \ldots, \mathcal{A}\left(v_{m}\right)\right\}$ for vertices $v_{1}, \ldots, v_{m}$ of $P$. As $P$ has $n+1$ vertices in a general position, $\widetilde{g}$ is diagonalizable over $\mathbb{R}$. Since $\tilde{g}: V \rightarrow V$ is a $\Gamma-$ module morphism, we obtain $\tilde{g}=\lambda I_{V}$ for $\lambda= \pm 1$ by Schur's lemma for $\mathbb{R}$.

One related question is:

Question 3.2 Can $\mathbb{D}(\widehat{P})$ for a compact hyperbolic Coxeter orbifold $\widehat{P}$ be compact and have dimension greater than or equal to 1 ?

This question was first asked by Benoist in 2005 as far as the authors know (see Marquis [40] for examples). 


\subsection{The reinterpretations of the deformation spaces as solution spaces}

Let $V$ be an $(n+1)$-dimensional real vector space. Denote by $\mathrm{M}_{s \times t}(\mathbb{R})$ the set of $s \times t$ matrices with real entries. We will identify $V$ and $V^{*}$ with $\mathrm{M}_{(n+1) \times 1}(\mathbb{R})=\mathbb{R}^{n+1}$ and $\mathrm{M}_{1 \times(n+1)}(\mathbb{R})=\left(\mathbb{R}^{n+1}\right)^{*}$ respectively as follows: we choose a basis $\left\{e_{1}, \ldots, e_{n+1}\right\}$ of $V$. Let $\left\{e_{1}^{*}, \ldots, e_{n+1}^{*}\right\}$ be the dual basis of $V^{*}$. If $\alpha_{i}=\alpha_{i, 1} e_{1}^{*}+\cdots+\alpha_{i, n+1} e_{n+1}^{*} \in V^{*}$, then $\alpha_{i}$ is identified with the $1 \times(n+1)$ matrix $\left(\alpha_{i, 1}, \ldots, \alpha_{i, n+1}\right)$. Similarly, if $b_{j}=b_{j, 1} e_{1}+\cdots+b_{j, n+1} e_{n+1} \in V$, then $b_{j}$ is identified with the $(n+1) \times 1$ matrix $\left(b_{j, 1}, \ldots, b_{j, n+1}\right)^{t}$, where $A^{t}$ means the transpose of a matrix $A$. Hence $\alpha_{i}\left(b_{j}\right)=\alpha_{i} b_{j}$, where the right-hand side is the scalar obtained as the matrix product of a $1 \times(n+1)$ matrix with an $(n+1) \times 1$ matrix. Denote by $I_{n+1}$ the $(n+1) \times(n+1)$ identity matrix. With this matrix notation, a reflection $R$ is of form $I_{n+1}-b \alpha$ for $\alpha \in V^{*}$ and $b \in V$ with $\alpha b=2$.

Let $\widehat{P}$ be a compact Coxeter $n$-orbifold with the fundamental chamber a properly convex $n$-polytope $P$ with $f$ facets in $\mathbb{S}^{n}$, and let $\mathbb{I}_{\widehat{P}}=\{1, \ldots, f\}$ be the index set of the facets. The orbifold structure of $\widehat{P}$ gives us the order $n_{i j}$ of the ridge $F_{i} \cap F_{j}$, $i, j \in \mathbb{I}_{\widehat{P}}, i \neq j$. Let $P$ be given by a system of linear inequalities $\alpha_{i} \geq 0\left(i \in \mathbb{I}_{\widehat{P}}\right)$ for $\alpha_{i}$ in $V^{*}$. Let $b_{i}$ be a vector with $\alpha_{i} b_{i}=2$ for each $i$, and let $R_{i}$ be the reflection $I_{n+1}-b_{i} \alpha_{i}$ for each $i \in \mathbb{I}$, and let $\Gamma \subset \mathrm{SL}_{n+1}^{ \pm}(\mathbb{R})$ be the group generated by the reflection $R_{i}$.

Define

$$
\begin{aligned}
& E_{1, \widehat{P}}=\left\{(i, j) \in \mathbb{I}_{\widehat{P}} \times \mathbb{I}_{\widehat{P}} \mid i=j\right\}, \\
& E_{2, \widehat{P}}=\left\{(i, j) \in \mathbb{I}_{\widehat{P}} \times \mathbb{I}_{\widehat{P}} \mid i<j, \quad F_{i} \text { and } F_{j} \text { are adjacent in } P \text { and } n_{i j}=2\right\}, \\
& E_{3, \widehat{P}}=\left\{(i, j) \in \mathbb{I}_{\widehat{P}} \times \mathbb{I}_{\widehat{P}} \mid i<j, \quad F_{i} \text { and } F_{j} \text { are adjacent in } P \text { and } n_{i j} \geq 3\right\}, \\
& E_{4, \widehat{P}}=\left\{(i, j) \in \mathbb{I}_{\widehat{P}} \times \mathbb{I}_{\widehat{P}} \mid i<j, \quad F_{i} \text { and } F_{j} \text { are not adjacent in } P\right\} .
\end{aligned}
$$

Vinberg's result leads us to solve the following system of polynomial equations:

- $a_{i i}=\alpha_{i} b_{i}=2$ for $(i, i) \in E_{1, \hat{P}}$.

- $a_{i j}=\alpha_{i} b_{j}=0$ and $a_{j i}=\alpha_{j} b_{i}=0$ for $(i, j) \in E_{2, \hat{P}}$.

- $a_{i j} a_{j i}=\alpha_{i} b_{j} \alpha_{j} b_{i}=4 \cos ^{2}\left(\frac{\pi}{n_{i j}}\right)$ for $(i, j) \in E_{3, \widehat{P}}$.

We call these polynomial equations Vinberg's equations. The $\alpha_{i}$ and $b_{i}$ are variables. Denote by $e$ the number of ridges and $e_{2}$ the number of ridges of order $2 . N_{\widehat{P}}=$ $f+e+e_{2}$ is the number of Vinberg's equations. Let $\left\{\Phi_{k}\right\}_{k}^{N_{\hat{P}}}$ be the set of polynomials in Vinberg's equations, and define a map

$$
\Phi_{\widehat{P}}:\left(V^{*}\right)^{f} \times V^{f} \rightarrow \mathbb{R}^{N_{\widehat{P}}}, \quad\left(\alpha_{1}, \ldots, \alpha_{f}, b_{1}, \ldots, b_{f}\right) \mapsto\left(\Phi_{1}, \ldots, \Phi_{N_{\widehat{P}}}\right) .
$$


Let $\mathbb{R}_{+}$be the set of positive real numbers. Denote by

$$
\theta:\left(\widetilde{G}:=\mathbb{R}_{+}^{f} \times \mathrm{SL}_{n+1}^{ \pm}(\mathbb{R})\right) \times\left(V^{*}\right)^{f} \times V^{f} \rightarrow\left(V^{*}\right)^{f} \times V^{f}
$$

the action given by

$$
\begin{aligned}
\left(d_{1}, \ldots, d_{f}, g\right) \cdot\left(\alpha_{1}, \ldots, \alpha_{f}, b_{1}, \ldots, b_{f}\right) & \\
& =\left(d_{1} \alpha_{1} g^{-1}, \ldots, d_{f} \alpha_{f} g^{-1}, d_{1}^{-1} g b_{1}, \ldots, d_{f}^{-1} g b_{f}\right),
\end{aligned}
$$

where $d_{i} \in \mathbb{R}_{+}$for each $i \in \mathbb{I}_{\widehat{P}}$ and $g \in \mathrm{SL}_{n+1}^{ \pm}(\mathbb{R})$. Then we have the invariance

$$
\Phi_{\widehat{P}} \circ \theta\left(d_{1}, \ldots, d_{f}, g\right)=\Phi_{\widehat{P}} .
$$

Define an open set

$$
\mathcal{U}_{\widehat{P}} \text { of elements }\left(\alpha_{1}, \ldots, \alpha_{f}, b_{1}, \ldots, b_{f}\right) \in\left(V^{*}\right)^{f} \times V^{f}
$$

that satisfy

- there exists $\vec{v} \neq O$ such that $\alpha_{i}(\vec{v})>0$ for each $i$,

- $\left\langle\alpha_{1}, \ldots, \alpha_{f}\right\rangle=V^{*}$,

- $a_{i j}<0$ and $a_{j i}<0$ if $(i, j) \in E_{3, \widehat{P}} \cup E_{4, \hat{P}}$,

- $a_{i j} a_{j i}>4$ if $(i, j) \in E_{4, \widehat{P}}$,

and where we replaced the condition (L2) with (L2)'.

We define the solution set

$$
\tilde{\mathbb{D}}(\widehat{P}):=\Phi_{\widehat{P}}^{-1}(0) \cap \mathcal{U}_{\widehat{P}}
$$

By invariance, $\widetilde{G}$ acts on $\mathcal{U}_{\widehat{P}}$ and on $\tilde{\mathbb{D}}(\widehat{P})$. Applying the action $\theta\left(d_{i}, g\right)$ on $\widetilde{\mathbb{D}}(\widehat{P})$, we have

$$
I_{n+1}-\left(d_{i}^{-1} g b_{i}\right)\left(d_{i} \alpha_{i} g^{-1}\right)=g\left(I_{n+1}-b_{i} \alpha_{i}\right) g^{-1}=g R_{i} g^{-1} \quad \text { for } i \in \mathbb{I}_{\widehat{P}} .
$$

So, the action $\theta\left(d_{1}, \ldots, d_{f}, g\right)$ on $\tilde{\mathbb{D}}(\widehat{P})$ corresponds to the conjugation in $\mathrm{SL}_{n+1}^{ \pm}(\mathbb{R})$. Define $\mathcal{M}$ as the submanifold of $\left(V^{*}\right)^{f} \times V^{f}$ of elements $\left(\alpha_{1}, \ldots, \alpha_{f}, b_{1}, \ldots, b_{f}\right)$, where $\alpha_{i} b_{i}=2$ for every $i=1, \ldots, f$. Define a map

$$
\mathcal{I}^{\prime}{ }_{\mathrm{SL}_{n+1}^{ \pm}}^{ \pm}(\mathbb{R}): \mathcal{M} \rightarrow R(n+1)^{f}
$$

by sending $\left(\alpha_{1}, \ldots, \alpha_{f}, b_{1}, \ldots, b_{f}\right)$ to $\left(r_{1}, \ldots, r_{f}\right)$ given by

$$
r_{i}(\cdot)=I_{V}-\alpha_{i}(\cdot) b_{i}: V \rightarrow V \quad \text { for each } i=1, \ldots, f .
$$

The map sends the information on the reflection subspace and the vertex to the reflection itself. Since a reflection is determined by its fixed-point subspace and the antipodal fixed 
point, the group $\mathbb{R}_{+}^{f} \times\left\{ \pm I_{V}\right\}^{f}$ acts simply transitively on the fibers of $\mathcal{I}_{\mathrm{SL}_{n+1}^{\prime}}^{ \pm}(\mathbb{R})$. Therefore, we obtain a principal fibration

$$
\begin{aligned}
& \mathbb{R}_{+}^{f} \times\left\{ \pm I_{V}\right\}^{f} \longrightarrow \mathcal{M} \\
& \downarrow_{\mathrm{SL}_{n+1}^{\prime}}^{\mathcal{I}^{\prime}} \\
& R(n+1)^{f} \text {. }
\end{aligned}
$$

Theorem 3.2 Let $\widehat{P}$ be a compact Coxeter $n$-orbifold. Assume that $\widehat{P}$ admits a real projective structure, but does not admit a spherical or Euclidean structure. We consider the solution set

$$
\tilde{\mathbb{D}}(\widehat{P}):=\Phi_{\widehat{P}}^{-1}(0) \cap \mathcal{U}_{\widehat{P}} \subset \mathcal{M}
$$

for Vinberg's equations $\Phi_{\widehat{P}}$.

- There exists a $\mathrm{PGL}_{n+1}(\mathbb{R})$-equivariant surjective map

$$
\mathcal{I}: \widetilde{\mathbb{D}}(\widehat{P}) /\left(\mathbb{R}_{+}^{f} \times\left\{ \pm I_{V}\right\}\right) \rightarrow D_{\text {rep }}(\widehat{P}) .
$$

- $D_{\text {rep }}(\widehat{P})$ is homeomorphic to $\tilde{\mathbb{D}}(\widehat{P}) /\left(\mathbb{R}_{+}^{f} \times\left\{ \pm I_{V}\right\}\right)$.

- The deformation space $\mathbb{D}(\hat{P})$ of real projective structures on the Coxeter orbifold $\widehat{P}$ is homeomorphic to a union of components of

$$
\tilde{\mathbb{D}}(\hat{P}) / \widetilde{G}=D_{\text {rep }}(\widehat{P}) / \operatorname{PGL}_{n+1}(\mathbb{R}), \quad \text { where } \widetilde{G}=\mathbb{R}_{+}^{f} \times \mathrm{SL}_{n+1}^{ \pm}(\mathbb{R}) .
$$

Proof The conditions of (3-4) imply that we have a nontrivial properly convex polytope as a fundamental chamber. Vinberg's equation, Theorem 2.1 and Proposition 2.3 imply that the image points are discrete faithful dividing reflection representations $\pi_{1}(\widehat{P}) \rightarrow \mathrm{SL}_{n+1}^{ \pm}(\mathbb{R})$.

Conversely, the collection of reflections generating the discrete faithful dividing reflection representation gives some point in $\widetilde{\mathbb{D}}(\widehat{P})$, ie in $\Phi_{\widehat{P}}^{-1}(0) \cap \mathcal{U}_{\widehat{P}}$, since it satisfies (L1) and (L2) ${ }^{\prime}$ as we showed in Section 2.2. Hence the map is surjective.

A representation given by assigning the fixed points and reflection facets to fundamental generators has ambiguity understood by (3-2). Thus, the fibers are again given as orbits of $\mathbb{R}_{+}^{f} \times\left\{ \pm I_{V}\right\}$, and $\mathcal{I}^{\prime} \mathrm{SL}_{n+1}^{ \pm}(\mathbb{R})$ restricts to a fibration $\tilde{\mathbb{D}}(\widehat{P}) \rightarrow D_{\text {rep }}(\widehat{P})$. The second item follows. The third item follows by Theorem 3.1 and the second item. 
Let $\mathbb{P} \mathbb{V}(\widehat{P})$ denote the space of $f \times f$ matrices $A=\left(a_{i j}\right)$ satisfying (L1) and (L2)' with $\operatorname{rank} A=n+1$ and no component of zero type. We recall from (2-1) that a diagonal matrix group $\mathbb{R}_{+}^{f}$ acts on $\mathbb{P V}(\widehat{P})$ by

$$
\left(d_{1}, \ldots, d_{f}\right) \circ\left(a_{i j}\right)=\left(d_{i} d_{j}^{-1} a_{i j}\right) .
$$

Corollary 3.1 Let $\widehat{P}$ be a compact Coxeter $n$-orbifold. Assume that $\widehat{P}$ admits a real projective structure, but does not admit a spherical or Euclidean structure. Then there exists a homeomorphism between each pair of the spaces below:

$$
\mathbb{D}(\widehat{P}) \longleftrightarrow D_{\text {rep }}(\widehat{P}) / \operatorname{PGL}_{n+1}(\mathbb{R}) \longleftrightarrow \tilde{\mathbb{D}}(\widehat{P}) / \widetilde{G} \longleftrightarrow \mathbb{P} \mathbb{V}(\widehat{P}) / \mathbb{R}_{+}^{f} .
$$

Proof Theorems 3.1 and 3.2 give the first and second correspondences. The map from the second one to the fourth one is obtained by going to the third one and taking $\alpha_{i}\left(b_{j}\right)$ as the entries of the Cartan matrices. Theorem 2.3 and Proposition 2.3 give us the map from the fourth one to the second one. These maps are inverses of each other by the uniqueness part of Theorem 2.3.

\section{Real projective structures near the hyperbolic structure}

We will obtain the information of real projective structures near the hyperbolic structure in terms of Zariski tangent spaces.

Recall in the previous section that real projective structures in the deformation space of a compact Coxeter orbifold $\widehat{P}$ correspond to solutions to Vinberg's equations. In Section 4.1 we study the Zariski tangent space to this solution space. In Section 4.2 we describe the space of hyperbolic structures of $\widehat{P}$ in terms of polynomial equations, forming so-called hyperbolic equations. In Section 4.3 we study the Zariski tangent space to the solution space of the hyperbolic equations. We compute the rank of the differential of the polynomial map from the hyperbolic equation in Proposition 4.1. In Section 4.5 we compare these two Zariski tangent spaces and combine this observation with the weak orderability of $\widehat{P}$ to prove Lemma 4.1, computing the rank of the differential of the polynomial map from Vinberg's equation. Finally, in Section 4.6, we prove the main result Theorem 4.1.

\subsection{The Zariski tangent space to Vinberg's equations}

Let $\widehat{P}$ be a Coxeter orbifold based on a properly convex $n$-polytope $P$ with $f$ facets in $\mathbb{S}^{n}$, and let $\mathbb{I}_{\hat{P}}=\{1, \ldots, f\}$ be the index set of the facets. Assume that $P$ is given by a system of linear inequalities, $\alpha_{i} \geq 0\left(i \in \mathbb{I}_{\hat{P}}\right)$ for $\alpha_{i} \in V^{*}$. Suppose that each $b_{i}$, $i=1, \ldots, f$, is a reflection vector with $\alpha_{i} b_{i}=2$. 
As in Section 3.2, we have variables $\alpha_{i} \in V^{*}=\left(\mathbb{R}^{n+1}\right)^{*}$ and $b_{i} \in V=\mathbb{R}^{n+1}$ for $i \in \mathbb{I}_{\widehat{P}}=\{1, \ldots, f\}$, and Vinberg's equations are of the following form:

- $\Phi_{i i}=\alpha_{i} b_{i}-2=0$ for $(i, i) \in E_{1, \widehat{P}}$.

- $\Phi_{i j}^{[1]}=\alpha_{i} b_{j}=0$ and $\Phi_{i j}^{[2]}=\alpha_{j} b_{i}=0$ for $(i, j) \in E_{2, \hat{P}}$.

- $\Phi_{i j}=\alpha_{i} b_{j} \alpha_{j} b_{i}-4 \cos ^{2}\left(\frac{\pi}{n_{i j}}\right)$ for $(i, j) \in E_{3, \widehat{P}}$.

Recall that $N_{\widehat{P}}$ is the number of Vinberg's equations, ie $N_{\widehat{\boldsymbol{P}}}=f+e+e_{2}$. Let

$$
\pi_{i}^{[1]}:\left(V^{*}\right)^{f} \times V^{f} \rightarrow V^{*} \text { and } \pi_{i}^{[2]}:\left(V^{*}\right)^{f} \times V^{f} \rightarrow V
$$

denote the projections onto the $i^{\text {th }}$ factor $V^{*}$ and the $(f+i)^{\text {th }}$ factor $V$, for every $i \in \mathbb{I}_{\widehat{\boldsymbol{P}}}$, respectively. For each $(i, j) \in E_{3, \widehat{P}}$, the derivative of $\Phi_{i j}$ at $p=$ $\left(\alpha_{1}, \ldots, \alpha_{f}, b_{1}, \ldots, b_{f}\right)$, considered as a linear map, is

$$
\begin{aligned}
D \Phi_{i j}(\dot{p}) & =a_{j i} \dot{\alpha}_{i} b_{j}+a_{i j} \dot{\alpha}_{j} b_{i}+a_{i j} \alpha_{j} \dot{b}_{i}+a_{j i} \alpha_{i} \dot{b}_{j} \\
& =a_{j i} \pi_{i}^{[1]}(\dot{p}) b_{j}+a_{i j} \pi_{j}^{[1]}(\dot{p}) b_{i}+a_{i j} \alpha_{j} \pi_{i}^{[2]}(\dot{p})+a_{j i} \alpha_{i} \pi_{j}^{[2]}(\dot{p})
\end{aligned}
$$

for $\dot{p}=\left(\dot{\alpha}_{1}, \ldots, \dot{\alpha}_{f}, \dot{b}_{1}, \ldots \dot{b}_{f}\right) \in\left(V^{*}\right)^{f} \times V^{f}$, and entries $a_{i j}$ of the Cartan matrix of $\widehat{P}$. Similarly, for each $(i, i) \in E_{1, \widehat{P}}$,

$$
D \Phi_{i i}(\dot{p})=\pi_{i}^{[1]}(\dot{p}) b_{i}+\alpha_{i} \pi_{i}^{[2]}(\dot{p}),
$$

and for each $(i, j) \in E_{2, \widehat{P}}$,

$$
D \Phi_{i j}^{[1]}(\dot{p})=\pi_{i}^{[1]}(\dot{p}) b_{j}+\alpha_{i} \pi_{j}^{[2]}(\dot{p}) \quad \text { and } \quad D \Phi_{i j}^{[2]}(\dot{p})=\pi_{j}^{[1]}(\dot{p}) b_{i}+\alpha_{j} \pi_{i}^{[2]}(\dot{p}) .
$$

More explicitly, combining Vinberg's equations gives a function $\Phi_{\widehat{P}}$ : $\left(V^{*}\right)^{f} \times V^{f} \rightarrow$ $\mathbb{R}^{N_{\widehat{P}}}$ and the rows of the $N_{\widehat{P}} \times 2(n+1) f$ Jacobian matrix $\left[D \Phi_{\widehat{P}}\right]$ are made up of $(n+1)$-entry blocks.

For each $(i, i) \in E_{1, \widehat{P}}$,

$$
\begin{aligned}
{\left[D \Phi_{i i}\right] } & =\left(0, \ldots, 0, b_{i, 1}, \ldots, b_{i, n+1}, 0, \ldots, 0, \alpha_{i, 1}, \ldots, \alpha_{i, n+1}, 0, \ldots, 0\right) \\
& =(0, \ldots, 0, \underbrace{b_{i}^{t}}_{i^{\text {th } b l o c k}}, 0, \ldots, 0, \underbrace{\alpha_{i}}_{(f+i)^{\text {th block }}}, 0, \ldots, 0) .
\end{aligned}
$$

For $(i, j) \in E_{2, \widehat{P}}$,

$\left[D \Phi_{i j}^{[1]}\right]$

$$
=(0, \ldots, 0, \underbrace{b_{j}^{t}}_{i^{\text {th }}}, 0, \ldots, 0, \underbrace{0}_{j^{\text {th }}}, 0, \ldots, 0, \underbrace{0}_{(f+i) \text { th }}, 0, \ldots, 0, \underbrace{\alpha_{i}}_{(f+j)^{\text {th }}}, 0, \ldots, 0),
$$


$\left[D \Phi_{i j}^{[2]}\right]$

$$
=(0, \ldots, 0, \underbrace{0}_{i^{\mathrm{th}}}, 0, \ldots, 0, \underbrace{b_{i}^{t}}_{j^{\mathrm{th}}}, 0, \ldots, 0, \underbrace{\alpha_{j}}_{(f+i)^{\mathrm{th}}}, 0, \ldots, 0, \underbrace{0}_{(f+j)^{\mathrm{th}}}, 0, \ldots, 0) .
$$

For $(i, j) \in E_{3, \hat{P}}$,

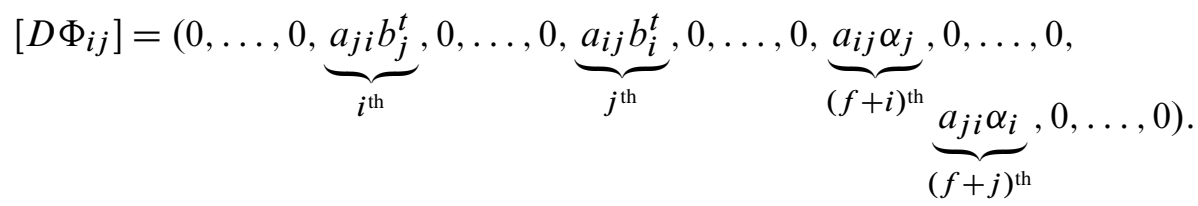

Suppose that $p$ is a point of $\Phi_{\widehat{p}}^{-1}(0)$. Then the Zariski tangent space at $p$ is the kernel of the Jacobian matrix $\left[D \Phi_{\hat{P}}\right]$ evaluated at $p$.

\subsection{The hyperbolic equations}

We let $V$ be an $(n+1)$-dimensional real vector space with coordinate functions $x_{1}, \ldots, x_{n+1}$, and let $\hat{P}$ be a compact hyperbolic Coxeter orbifold with the fundamental chamber equal to a compact $n$-polytope $P$ in the Klein projective model of the $n-$ dimensional hyperbolic space $\mathbb{H}^{n}$. Let $P$ have facets $F_{i}$ for $i \in \mathbb{I}_{\widehat{P}}=\{1,2, \ldots, f\}$.

Denote by $v_{i} \in V$ the inward unit normal to the subspace spanned by vectors in directions of $F_{i}$ with respect to the Lorentzian inner product on $V$. Then the following system of linear inequalities define $P$ :

$$
\left\langle v_{i}, x\right\rangle \geq 0 \quad \text { for each } i \in \mathbb{I}_{\widehat{P}} \quad \text { and } \quad x_{1}=1 .
$$

To construct a hyperbolic Coxeter $n$-polytope $P$ with prescribed dihedral angles $\pi / n_{i j}$, we need to solve the equations

$$
\begin{aligned}
& \left\langle v_{i}, v_{i}\right\rangle=1 \quad \text { for each } i \in \mathbb{I}_{\widehat{P}}, \\
& \left\langle v_{i}, v_{j}\right\rangle=-\cos \left(\frac{\pi}{n_{i j}}\right) \quad \text { if facets } F_{i} \text { and } F_{j} \text { are adjacent in } P .
\end{aligned}
$$

We call these equations hyperbolic equations. To compare the hyperbolic equations with Vinberg's equations, the system of linear inequalities defining $P$ is given by

$$
\alpha_{i}(x) \geq 0 \quad \text { for } i \in \mathbb{I}_{\widehat{P}} \quad \text { and } \quad x_{1}=1, x \in V,
$$

where the linear functional $\alpha_{i} \in V^{*}$ is given by $\alpha_{i}(v)=2\left\langle v_{i}, v\right\rangle$. The hyperbolic reflection in the facet $F_{i}$ is a map

$$
R_{i}(v)=v-2\left\langle v_{i}, v\right\rangle v_{i}=v-\alpha_{i}(v) b_{i}
$$


for $b_{i}=v_{i}$. Thus taking $\alpha_{i}=2\left\langle v_{i}, \cdot\right\rangle$ and $b_{i}=v_{i}$ gives a hyperbolic point $t$ in the space $\Phi_{\widehat{P}}^{-1}(0)$ corresponding to the hyperbolic structure on $\widehat{P}$. We rewrite the equation in another way. If facets $F_{i}$ and $F_{j}$ are adjacent in $P$, then

$$
a_{i j}=\alpha_{i}\left(b_{j}\right)=2\left\langle v_{i}, v_{j}\right\rangle=-2 \cos \left(\frac{\pi}{n_{i j}}\right)
$$

and thus

$$
\begin{aligned}
a_{i i}=2\left\langle v_{i}, v_{i}\right\rangle=2 & \text { for }(i, i) \in E_{1, \widehat{P}}, \\
a_{i j}=0 \quad \text { and } \quad a_{j i}=0 & \text { for }(i, j) \in E_{2, \widehat{P}}, \\
a_{i j} a_{j i}=4 \cos ^{2}\left(\frac{\pi}{n_{i j}}\right) & \text { for }(i, j) \in E_{3, \widehat{P}} .
\end{aligned}
$$

\subsection{The Zariski tangent space to the hyperbolic equations}

As in Section 4.2, we assume that $P$ is a compact hyperbolic Coxeter $n$-polytope where the dihedral angle at each ridge $F_{i j}=F_{i} \cap F_{j}$ equals $\pi / n_{i j}$ for an integer $n_{i j} \geq 2$. Constructing such a hyperbolic $n$-polytope $P$ is the same as solving the system of hyperbolic equations (4-2) for the unit normals $v_{i}$. Equivalently we can write these equations in terms of the reflection vectors $b_{i}=v_{i}$. This gives the following system of $m=f+e$ equations:

$$
\begin{array}{ll}
\Psi_{i i}=2\left\langle b_{i}, b_{i}\right\rangle-2=0 & \text { for }(i, i) \in E_{1, \hat{P}}, \\
\Psi_{i j}=2\left\langle b_{i}, b_{j}\right\rangle+2 \cos \left(\frac{\pi}{n_{i j}}\right)=0 & \text { for }(i, j) \in E_{2, \widehat{P}} \cup E_{3, \widehat{P}} .
\end{array}
$$

Combining these gives a function $\Psi_{\widehat{P}}: V^{f}=\mathbb{R}^{(n+1) f} \rightarrow \mathbb{R}^{m}$, and $\Psi_{\widehat{P}}^{-1}(0)$ contains Coxeter $n$-polytopes in $\mathbb{H}^{n}$ with the desired dihedral angles.

We define an open manifold

$$
\text { (4-4) } \mathcal{W}_{\widehat{P}}:=\left\{\left(b_{1}, \ldots, b_{f}\right) \in V^{f} \mid\left\langle b_{i}, b_{i}\right\rangle=1, i \in \mathbb{I}_{\widehat{P}},\left\langle b_{i}, b_{j}\right\rangle<-2 \text { if }(i, j) \in E_{4, \hat{P}}\right\}
$$

The $f$-tuple $\left(b_{1}, \ldots, b_{f}\right)$ of normal vectors to facets for a compact hyperbolic polytope satisfies (4-3) and is in $\mathcal{W}_{\widehat{P}}$ (see Roeder, Hubbard and Dunbar [43]).

Now we compute the derivative $D \Psi_{\widehat{P}}$ at a hyperbolic point $t$. Setting $\alpha_{i}=2\left\langle v_{i}, \cdot\right\rangle$, $i=1, \ldots, f$, to be the linear functionals defining the facets of $P$, we obtain

$$
D \Psi_{i j}(\dot{b})=2\left\langle\dot{b}_{i}, b_{j}\right\rangle+2\left\langle b_{i}, \dot{b}_{j}\right\rangle=\alpha_{j} \dot{b}_{i}+\alpha_{i} \dot{b}_{j}
$$

for $\dot{b}:=\left(\dot{b}_{1}, \ldots, \dot{b}_{f}\right) \in V^{f}, \dot{b}_{i} \in V, i=1, \ldots, f$. For $i=j$, this becomes

$$
D \Psi_{i i}(\dot{b})=2 \alpha_{i} \dot{b}_{i} \quad \text { for } \dot{b}=\left(\dot{b}_{1}, \ldots, \dot{b}_{f}\right) \in V^{f} .
$$

Equivalently, the rows of the $m \times(n+1) f$ Jacobian matrix [ $D \Psi_{\hat{P}}$ consist of blocks, each consisting of $(n+1)$ entries. 
For each $(i, i) \in E_{1, \widehat{P}}$,

$$
\begin{aligned}
{\left[D \Psi_{i i}\right] } & =\left(0, \ldots, 0,2 \alpha_{i, 1}, \ldots, 2 \alpha_{i, n+1}, 0, \ldots, 0\right) \\
& =(0, \ldots, 0, \underbrace{2 \alpha_{i}}_{i \text { th block }}, 0, \ldots, 0)
\end{aligned}
$$

and for each $(i, j) \in E_{2, \widehat{P}} \cup E_{3, \widehat{P}}$,

$$
\left[D \Psi_{i j}\right]=(0, \ldots, 0, \underbrace{\alpha_{j}}_{i \text { th block }}, 0, \ldots, 0, \underbrace{\alpha_{i}}_{j \text { th block }}, 0, \ldots, 0) .
$$

Then the Zariski tangent space to $\Psi_{\widehat{P}}^{-1}(0) \cap \mathcal{W}_{\widehat{P}}$ at $t$ is ker $D \Psi_{\widehat{P}}$.

Recall that $\operatorname{Hom}\left(\pi_{1}(\widehat{P}), \operatorname{PO}(1, n)\right)$ is an algebraic subset of the space $\operatorname{PO}(1, n)^{f}$, where $f$ is the number of fundamental generators, ie the number of facets of $P$. We give it the standard subspace topology.

Proposition 4.1 Let $P$ be a compact hyperbolic Coxeter $n$-polytope. Suppose that $\widehat{P}$ is the Coxeter orbifold arising from $P$, with the associated holonomy representation $h_{0}$, and let $\bar{b}_{0}$ denote the $f$-tuple of vectors normal to the facets of $P$ in the Lorentzian spaces. Then we have the following.

- The orbit of $h_{0}$ under $\mathrm{PO}(1, n)$ contains an open neighborhood of $h_{0}$ in

$$
\operatorname{Hom}\left(\pi_{1}(\widehat{P}), \operatorname{PO}(1, n)\right)
$$

and this is a smooth $\frac{n(n+1)}{2}-$ manifold in a neighborhood of $h_{0}$.

- A neighborhood of $\bar{b}_{0}$ at $\Psi_{\widehat{P}}^{-1}(0)$ is diffeomorphic to a neighborhood of $h_{0}$ in the real algebraic set $\operatorname{Hom}\left(\pi_{1}(\widehat{P}), \mathrm{PO}(1, n)\right)$.

- $\operatorname{dim} \operatorname{ker} D \Psi_{\widehat{P}, \bar{b}_{0}}=\operatorname{dim} \mathfrak{s o}(1, n)=\frac{n(n+1)}{2}$.

Proof Let $\pi_{1}(\widehat{P})$ act on the Lie algebra $\mathfrak{s o}(1, n)$ of $\mathrm{PO}(1, n)$ by the representation Ad $\circ h_{0}$. By the work of Weil [50], the Zariski tangent space to $\operatorname{Hom}\left(\pi_{1}(\widehat{P}), \operatorname{PO}(1, n)\right)$ at $h_{0}$ is isomorphic to the vector space $Z^{1}\left(\pi_{1}(\widehat{P}), \mathfrak{s o}(1, n)_{\mathrm{Ad} \circ h_{0}}\right)$ of 1 -cocycles for computing the group cohomology; see also Raghunathan [42, Chapters 6 and 7] and Goldman [30, Section 1] for a material on cycles and cocycles.

A neighborhood of $\operatorname{Hom}\left(\pi_{1}(\widehat{P}), \mathrm{PO}(1, n)\right)$ of $h_{0}$ consists of holonomies of hyperbolic Coxeter orbifolds diffeomorphic to $\widehat{P}$ by Choi [16, Theorem 1]. The Mostow rigidity shows that a neighborhood of $h_{0}$ in $\operatorname{Hom}\left(\pi_{1}(\hat{P}), \operatorname{PO}(1, n)\right)$ is inside the orbit of $h_{0}$ under the conjugation action of $\mathrm{PO}(1, n)$. The orbit is a smooth $\frac{1}{2} n(n+1)$-manifold in a neighborhood of $h_{0}$ by an easy real algebraic group action theory argument since 
the hyperbolic holonomy group $h_{0}\left(\pi_{1}(\widehat{P})\right)$ has a trivial centralizer in $\mathrm{PO}(1, n)$. This proves the first item.

Let $R(1, n)$ denote the subspace of $\mathrm{PO}(1, n)$ of reflections fixing a hyperplane meeting the positive cone, and

$$
\mathcal{U}^{f}:=\left\{\left(b_{1}, \ldots, b_{f}\right) \in V^{f} \mid\left\langle b_{i}, b_{i}\right\rangle=1, i \in \mathbb{I}_{\widehat{P}}\right\},
$$

which is a smooth manifold. Define the map

$$
\mathcal{I}^{\prime}{ }_{\mathrm{PO}(1, n)}: \mathcal{U}^{f} \rightarrow R(1, n)^{f}
$$

by sending $\left(b_{1}, \ldots, b_{f}\right)$ to $\left(r_{1}, \ldots, r_{f}\right)$ such that

$$
r_{i}(\cdot)=I_{V}-2\left\langle b_{i}, \cdot\right\rangle b_{i}, \quad i=1, \ldots, f .
$$

Here, $\left\{ \pm I_{V}\right\}^{f}$ acts on fibers transitively and the map is a covering map. Consider the restriction

$$
\mathcal{I}^{\prime \prime}: \Psi_{\widehat{P}}^{-1}(0) \cap \mathcal{W}_{\widehat{P}} \subset \mathcal{W}_{\widehat{P}} \rightarrow \operatorname{Hom}\left(\pi_{1}(\widehat{P}), \operatorname{PO}(1, n)\right) \subset R(1, n)^{f},
$$

where $\mathcal{W}_{\widehat{P}}$ is an open subset of $\mathcal{U}^{f}$. The relations defining

$$
\Psi_{\widehat{P}}^{-1}(0) \quad \text { and } \operatorname{Hom}\left(\pi_{1}(\hat{P}), \operatorname{PO}(1, n)\right)
$$

coincide under $\mathcal{I}^{\prime \prime}$ and the above restriction $\mathcal{I}^{\prime \prime}$ of $\mathcal{I}_{\mathrm{PO}(1, n)}^{\prime}$ is a local diffeomorphism to its image. Here, $\left\{ \pm I_{V}\right\}$ acts transitively on fibers. This proves the second item. (We are in the situation of diffeomorphic coordinate variable changes, heuristically speaking.)

We also obtain

$$
\operatorname{dim} \operatorname{ker} D \Psi_{\widehat{P}, \bar{b}_{0}}=\operatorname{dim} Z^{1}\left(\pi_{1}(\widehat{P}), \mathfrak{s o}(1, n)_{\mathrm{Ad} \circ h_{0}}\right)
$$

since the second Zariski tangent space is again given by a system of algebraic equations on $R(1, n)^{f}$. By Weil infinitesimal rigidity [49], $H^{1}\left(\pi_{1}(\widehat{P}), \mathfrak{s o}(1, n)_{\mathrm{Ad} \circ h_{0}}\right)=0$, and it follows that

$$
\operatorname{dim} Z^{1}\left(\pi_{1}(\widehat{P}), \mathfrak{s o}(1, n)_{\mathrm{Ad} \circ h_{0}}\right)=\operatorname{dim} B^{1}\left(\pi_{1}(\widehat{P}), \mathfrak{s o}(1, n)_{\mathrm{Ad} \circ h_{0}}\right) .
$$

Since $\operatorname{PO}(1, n)$ acts freely on $\operatorname{Hom}\left(\pi_{1}(\widehat{P}), \operatorname{PO}(1, n)\right)$ with smooth orbits, the dimension $\operatorname{dim} B^{1}\left(\pi_{1}(\hat{P}), \operatorname{so}(1, n)_{\mathrm{Ad} \circ h_{0}}\right)$ of the tangent space of the orbit passing $h_{0}$ is $\operatorname{dim} \mathfrak{s o}(1, n)=(n(n+1)) / 2$. This proves the third item; see also the proof of Choi [20, Theorem 1]. 


\subsection{The main theorem}

Definition 4.1 A real projective Coxeter $n$-orbifold $\widehat{P}$ is weakly orderable if the facets of the fundamental polytope $P$ in $\mathbb{S}^{n}$ can be labeled by integers $\{1, \ldots, f\}$ so that for each facet $F_{i}$,

- the cardinality of the collection

$$
\mathcal{F}_{i}:=\left\{F_{j} \mid j>i \text { and the ridge } F_{i} \cap F_{j} \text { has order } 2\right\}
$$

is less than or equal to $n$,

- the collection $\mathcal{F}_{i}$ is in general position whenever $\mathcal{F}_{i}$ is not empty.

Here, the general position for a collection of facets means that the defining linear equations of the facets are linearly independent. For $n=3$, we automatically have the last general position condition by Choi [20, Lemma 3]. Thus, the second definition generalizes the earlier definition for $n=3$.

Recall that a $n$-polytope $P$ in $\mathbb{S}^{n}$ is simple if exactly $n$ facets meet at each vertex. Let $f$ and $e$ be the numbers of facets and ridges of $P$ respectively. We introduce an integer

$$
\delta_{P}=e-n f+\frac{1}{2} n(n+1)
$$

which depends only on the polytope $P$ but not on the orbifold structure. Barnette [2] showed for simple polytopes $P$ that $\delta_{P} \geq 0$; see also Greene [32]. In our context, $\delta_{P}=0$ indicates the full rank property of hyperbolic equations; see (4-8).

Theorem 4.1 Let $P$ be a compact hyperbolic Coxeter $n$-polytope, and suppose that $\widehat{P}$ is the Coxeter orbifold arising from $P$. Suppose that

(C1) $\delta_{P}=0$,

(C2) $\widehat{P}$ is weakly orderable.

Then a neighborhood of the hyperbolic point in $\mathbb{D}(\widehat{P})$ is homeomorphic to a cell of dimension $e_{+}(\widehat{P})-n$.

\subsection{The main lemma}

The proof of Lemma 4.1 is technical, hence in Example 4.1 we will introduce a simple example to explain the procedure. 
Lemma 4.1 Let $P$ be a compact hyperbolic Coxeter $n$-polytope, and suppose that $\widehat{P}$ is the Coxeter orbifold arising from $P$. Let $e_{2}$ be the number of ridges of order 2, and let $\bar{b}_{0} \in V^{f}$ be the $f$-tuple $\left(b_{1}, \ldots, b_{f}\right)$ of normal unit vectors for facets of $P$, and $\bar{\alpha}_{0} \in V^{* f}$ the $f$-tuple $\left(\alpha_{1}, \ldots, \alpha_{f}\right)$ of dual vectors $\alpha_{i}=2\left\langle b_{i}, \cdot\right\rangle$. If $\hat{P}$ is weakly orderable, then

$$
\operatorname{rank} D \Phi_{\widehat{P},\left(\bar{\alpha}_{0}, \bar{b}_{0}\right)}=\operatorname{rank} D \Psi_{\widehat{P}, \bar{b}_{0}}+e_{2}
$$

Proof Since $\widehat{P}$ is weakly orderable, we order the facets of $P$ so that each facet contains at most $n$ ridges of order 2 in facets of higher indices. Define $\mathbb{I}_{\widehat{P}}(k)=\left\{i \in \mathbb{I}_{\widehat{\boldsymbol{P}}} \mid i>k\right.$ and $F_{i} \cap F_{k}$ is a ridge of order 2$\} \quad$ and $\quad i(k)=\left|\mathbb{I}_{\widehat{\boldsymbol{P}}}(k)\right|$.

The set can be empty and $i(k)=0$. We may enumerate

$$
\mathbb{I}_{\widehat{P}}(k)=\left\{\mathbb{I}_{\widehat{P}}(k, 1), \ldots, \mathbb{I}_{\widehat{P}}(k, i(k))\right\}
$$

such that if $s<t$, then $\mathbb{I}_{\widehat{P}}(k, s)<\mathbb{I}_{\hat{P}}(k, t)$. Clearly,

$$
k<\mathbb{I}_{\widehat{P}}(k, l) \text { for } 1 \leq l \leq i(k) .
$$

That is,

$$
\begin{array}{r}
1<\mathbb{I}_{\widehat{P}}(1)=\left\{\mathbb{I}_{\widehat{P}}(1,1)<\mathbb{I}_{\widehat{P}}(1,2)<\cdots<\mathbb{I}_{\widehat{P}}(1, i(1))\right\}, \\
2<\mathbb{I}_{\widehat{P}}(2)=\left\{\mathbb{I}_{\widehat{P}}(2,1)<\mathbb{I}_{\widehat{P}}(2,2)<\cdots<\mathbb{I}_{\widehat{P}}(2, i(2))\right\}, \\
\vdots \\
q<\mathbb{I}_{\widehat{P}}(q)=\left\{\mathbb{I}_{\widehat{P}}(q, 1)<\mathbb{I}_{\widehat{P}}(q, 2)<\cdots<\mathbb{I}_{\widehat{P}}(q, i(q))\right\},
\end{array}
$$

for some $q, 1 \leq q<f$. Then we have

$$
\begin{aligned}
E_{2, \widehat{P}}=\left\{\left(1, \mathbb{I}_{\widehat{P}}(1,1)\right),\left(1, \mathbb{I}_{\widehat{P}}(1,2)\right), \ldots,\left(1, \mathbb{I}_{\widehat{P}}(1, i(1))\right),\right. \\
\left(2, \mathbb{I}_{\widehat{P}}(2,1)\right),\left(2, \mathbb{I}_{\widehat{P}}(2,2)\right), \ldots,\left(2, \mathbb{I}_{\widehat{P}}(2, i(2))\right), \\
\vdots \\
\left.\left(q, \mathbb{I}_{\widehat{P}}(q, 1)\right),\left(q, \mathbb{I}_{\widehat{P}}(q, 2)\right), \ldots,\left(q, \mathbb{I}_{\widehat{P}}(q, i(q))\right)\right\},
\end{aligned}
$$

where $i(k) \leq n$. We note that

$$
\sum_{k=1}^{q} i(k)=\left|E_{2, \widehat{P}}\right|=e_{2} .
$$


Define the $1 \times(n+1) f$ matrices

$$
\alpha_{[i]}^{[j]}=(0, \ldots, 0, \underbrace{\alpha_{i}}_{j \text { th block }}, 0, \ldots, 0) \quad \text { and } b_{[i]}^{[j]}=(0, \ldots, 0, \underbrace{b_{i}^{t}}_{j \text { th block }}, 0, \ldots, 0) .
$$

Denote by $J$ the $(n+1) \times(n+1)$-diagonal matrix with diagonal entries $-1,1, \ldots, 1$. (We will now omit from $D \Phi_{i j,(\bar{\alpha}, \bar{b})}$ the subscripts $(\bar{\alpha}, \bar{b})$ to simplify.)

We note that $\alpha_{i}=2 b_{i}^{t} J$ and $a_{i j}=a_{i j}$ at the hyperbolic point by Vinberg [47, Proposition 24] and the rows of the $N_{\widehat{P}} \times 2(n+1) f$ matrix $\left[D \Phi_{\widehat{P}}\right]$ are as follows:

$$
\begin{aligned}
{\left[D \Phi_{i i}\right] } & =\left(b_{[i]}^{[i]}, \alpha_{[i]}^{[i]}\right) & & (i, i) \in E_{1, \hat{P}}, \\
{\left[D \Phi_{i j}^{[1]}\right] } & =\left(b_{[j]}^{[i]}, \alpha_{[i]}^{[j]}\right) & & (i, j) \in E_{2, \widehat{P}}, \\
{\left[D \Phi_{i j}^{[2]}\right] } & =\left(b_{[i]}^{[j]}, \alpha_{[j]}^{[i]}\right) & & (i, j) \in E_{2, \widehat{P}}, \\
{\left[D \Phi_{i j}\right] } & =\left(a_{i j} b_{[i]}^{[j]}+a_{j i} b_{[j]}^{[i]}, a_{j i} \alpha_{[i]}^{[j]}+a_{i j} \alpha_{[j]}^{[i]}\right) & & (i, j) \in E_{3, \hat{P}},
\end{aligned}
$$

by (4-1). (Here, we merely indicate the rows and not write the whole matrix.)

Before completing the proof, let us give an example to illustrate.

Example 4.1 As an example, we use a compact 3-dimensional hyperbolic tetrahedron to illustrate the method in the proof of Lemma 4.1; see Figure 1. Here, if an edge is labeled $l$, then its dihedral angle is $\pi / l$. We will simply use the inherited notation here with obvious meaning.

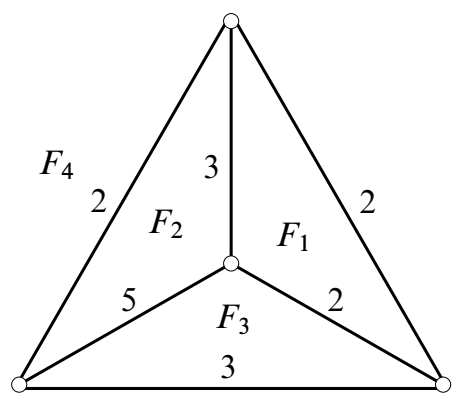

Figure 1: A compact hyperbolic tetrahedron

Then

$$
\begin{gathered}
\mathbb{I}_{\widehat{P}}(1)=\left\{\mathbb{I}_{\widehat{P}}(1,1)=3<\mathbb{I}_{\widehat{P}}(1,2)=4\right\}, \quad \mathbb{I}_{\widehat{P}}(2)=\left\{\mathbb{I}_{\widehat{P}}(2,1)=4\right\}, \\
E_{2, \widehat{P}}=\{(1,3),(1,4),(2,4)\}, \quad E_{3, \widehat{P}}=\{(1,2),(2,3),(3,4)\}, \\
E_{1, \widehat{P}}=\{(1,1),(2,2),(3,3),(4,4)\},
\end{gathered}
$$


and hence

$$
\begin{aligned}
& {\left[D \Phi_{\hat{P}}\right]=\left[\begin{array}{cc}
D \Phi_{i j}^{[1]} & (i, j) \in E_{2, \widehat{P}} \\
D \Phi_{i j}^{[2]} & (i, j) \in E_{2, \widehat{P}} \\
D \Phi_{i j} & (i, j) \in E_{3, \widehat{P}} \\
D \Phi_{i i} & (i, i) \in E_{1, \widehat{P}}
\end{array}\right]=\left[\begin{array}{c}
D \Phi_{13}^{[1]} \\
D \Phi_{14}^{[1]} \\
D \Phi_{24}^{[1]} \\
\hline D \Phi_{13}^{[2]} \\
D \Phi_{14}^{[2]} \\
D \Phi_{24}^{[2]} \\
D \Phi_{12} \\
D \Phi_{23} \\
D \Phi_{34} \\
D \Phi_{11} \\
D \Phi_{22} \\
D \Phi_{33} \\
D \Phi_{44}
\end{array}\right]} \\
& =\left[\begin{array}{llll|llll}
b_{3}^{t} & 0 & 0 & 0 & 0 & 0 & \alpha_{1} & 0 \\
b_{4}^{t} & 0 & 0 & 0 & 0 & 0 & 0 & \alpha_{1} \\
0 & b_{4}^{t} & 0 & 0 & 0 & 0 & 0 & \alpha_{2} \\
\hline 0 & 0 & b_{1}^{t} & 0 & \alpha_{3} & 0 & 0 & 0 \\
0 & 0 & 0 & b_{1}^{t} & \alpha_{4} & 0 & 0 & 0 \\
0 & 0 & 0 & b_{2}^{t} & 0 & \alpha_{4} & 0 & 0 \\
\hline a_{21} b_{2}^{t} & a_{12} b_{1}^{t} & 0 & 0 & a_{12} \alpha_{2} & a_{21} \alpha_{1} & 0 & 0 \\
0 & a_{32} b_{3}^{t} & a_{23} b_{2}^{t} & 0 & 0 & a_{23} \alpha_{3} & a_{32} \alpha_{2} & 0 \\
0 & 0 & a_{43} b_{4}^{t} & a_{34} b_{3}^{t} & 0 & 0 & a_{34} \alpha_{4} & a_{43} \alpha_{3} \\
b_{1}^{t} & 0 & 0 & 0 & \alpha_{1} & 0 & 0 & 0 \\
0 & b_{2}^{t} & 0 & 0 & 0 & \alpha_{2} & 0 & 0 \\
0 & 0 & b_{3}^{t} & 0 & 0 & 0 & \alpha_{3} & 0 \\
0 & 0 & 0 & b_{4}^{t} & 0 & 0 & 0 & \alpha_{4}
\end{array}\right]
\end{aligned}
$$

where 0 is the zero $1 \times 4$ matrix. 
First, for each $(i, j) \in E_{2, \widehat{P}}$, add a row $\left[D \Phi_{i j}^{[1]}\right]$ of $\left[D \Phi_{\widehat{P}}\right]$ to another row $\left[D \Phi_{i j}^{[2]}\right]$. This gives

$\left[\begin{array}{cccc|cccc}b_{3}^{t} & 0 & 0 & 0 & 0 & 0 & \alpha_{1} & 0 \\ b_{4}^{t} & 0 & 0 & 0 & 0 & 0 & 0 & \alpha_{1} \\ 0 & b_{4}^{t} & 0 & 0 & 0 & 0 & 0 & \alpha_{2} \\ \hline \boldsymbol{b}_{3}^{t} & 0 & b_{1}^{t} & 0 & \alpha_{3} & 0 & \alpha_{1} & 0 \\ \boldsymbol{b}_{4}^{t} & 0 & 0 & b_{1}^{t} & \alpha_{4} & 0 & 0 & \alpha_{1} \\ 0 & \boldsymbol{b}_{4}^{t} & 0 & b_{2}^{t} & 0 & \alpha_{4} & 0 & \alpha_{2} \\ \hline a_{21} b_{2}^{t} & a_{12} b_{1}^{t} & 0 & 0 & a_{12} \alpha_{2} & a_{21} \alpha_{1} & 0 & 0 \\ 0 & a_{32} b_{3}^{t} & a_{23} b_{2}^{t} & 0 & 0 & a_{23} \alpha_{3} & a_{32} \alpha_{2} & 0 \\ 0 & 0 & a_{43} b_{4}^{t} & a_{34} b_{3}^{t} & 0 & 0 & a_{34} \alpha_{4} & a_{43} \alpha_{3} \\ b_{1}^{t} & 0 & 0 & 0 & \alpha_{1} & 0 & 0 & 0 \\ 0 & b_{2}^{t} & 0 & 0 & 0 & \alpha_{2} & 0 & 0 \\ 0 & 0 & b_{3}^{t} & 0 & 0 & 0 & \alpha_{3} & 0 \\ 0 & 0 & 0 & b_{4}^{t} & 0 & 0 & 0 & \alpha_{4}\end{array}\right]$.

Second, for $(i, j) \in E_{3, \hat{P}}$, multiply a row $\left[D \Phi_{i j}\right]$ of $\left[D \Phi_{\hat{P}}\right]$ by $a_{i j}^{-1}$ :

$\left[\begin{array}{cccc|cccc}b_{3}^{t} & 0 & 0 & 0 & 0 & 0 & \alpha_{1} & 0 \\ b_{4}^{t} & 0 & 0 & 0 & 0 & 0 & 0 & \alpha_{1} \\ 0 & b_{4}^{t} & 0 & 0 & 0 & 0 & 0 & \alpha_{2} \\ \hline b_{3}^{t} & 0 & b_{1}^{t} & 0 & \alpha_{3} & 0 & \alpha_{1} & 0 \\ b_{4}^{t} & 0 & 0 & b_{1}^{t} & \alpha_{4} & 0 & 0 & \alpha_{1} \\ 0 & b_{4}^{t} & 0 & b_{2}^{t} & 0 & \alpha_{4} & 0 & \alpha_{2} \\ \hline \boldsymbol{b}_{2}^{t} & \boldsymbol{b}_{1}^{t} & 0 & 0 & \alpha_{2} & \alpha_{1} & 0 & 0 \\ 0 & \boldsymbol{b}_{3}^{t} & \boldsymbol{b}_{2}^{t} & 0 & 0 & \alpha_{3} & \alpha_{2} & 0 \\ 0 & 0 & \boldsymbol{b}_{4}^{t} & \boldsymbol{b}_{3}^{t} & 0 & 0 & \alpha_{4} & \alpha_{3} \\ b_{1}^{t} & 0 & 0 & 0 & \alpha_{1} & 0 & 0 & 0 \\ 0 & b_{2}^{t} & 0 & 0 & 0 & \alpha_{2} & 0 & 0 \\ 0 & 0 & b_{3}^{t} & 0 & 0 & 0 & \alpha_{3} & 0 \\ 0 & 0 & 0 & b_{4}^{t} & 0 & 0 & 0 & \alpha_{4}\end{array}\right]$


Third, for each $(i, i) \in E_{1, \widehat{P}}$, multiply a row $\left[D \Phi_{i i}\right]$ of $\left[D \Phi_{\hat{P}}\right]$ by 2 :

$$
\left[\begin{array}{cccc|cccc}
b_{3}^{t} & 0 & 0 & 0 & 0 & 0 & \alpha_{1} & 0 \\
b_{4}^{t} & 0 & 0 & 0 & 0 & 0 & 0 & \alpha_{1} \\
0 & b_{4}^{t} & 0 & 0 & 0 & 0 & 0 & \alpha_{2} \\
\hline b_{3}^{t} & 0 & b_{1}^{t} & 0 & \alpha_{3} & 0 & \alpha_{1} & 0 \\
b_{4}^{t} & 0 & 0 & b_{1}^{t} & \alpha_{4} & 0 & 0 & \alpha_{1} \\
0 & b_{4}^{t} & 0 & b_{2}^{t} & 0 & \alpha_{4} & 0 & \alpha_{2} \\
\hline b_{2}^{t} & b_{1}^{t} & 0 & 0 & \alpha_{2} & \alpha_{1} & 0 & 0 \\
0 & b_{3}^{t} & b_{2}^{t} & 0 & 0 & \alpha_{3} & \alpha_{2} & 0 \\
0 & 0 & b_{4}^{t} & b_{3}^{t} & 0 & 0 & \alpha_{4} & \alpha_{3} \\
\mathbf{2} b_{1}^{t} & 0 & 0 & 0 & \mathbf{2} \alpha_{1} & 0 & 0 & 0 \\
0 & \mathbf{2} b_{2}^{t} & 0 & 0 & 0 & \mathbf{2} \alpha_{2} & 0 & 0 \\
0 & 0 & \mathbf{2} b_{3}^{t} & 0 & 0 & 0 & \mathbf{2} \alpha_{3} & 0 \\
0 & 0 & 0 & \mathbf{2} b_{4}^{t} & 0 & 0 & 0 & \mathbf{2} \alpha_{4}
\end{array}\right] .
$$

Fourth, multiply the left 16 columns of $\left[D \Phi_{\hat{P}}\right]$ by 2 and the $(4 i-3)^{\text {th }}$ columns $\left(i \in \mathbb{I}_{\hat{P}}=\{1,2,3,4\}\right)$ of $\left[D \Phi_{\hat{P}}\right]$ by -1 respectively:

$$
\left[\begin{array}{cccc|cccc}
\boldsymbol{\alpha}_{3} & 0 & 0 & 0 & 0 & 0 & \alpha_{1} & 0 \\
\boldsymbol{\alpha}_{4} & 0 & 0 & 0 & 0 & 0 & 0 & \alpha_{1} \\
0 & \boldsymbol{\alpha}_{4} & 0 & 0 & 0 & 0 & 0 & \alpha_{2} \\
\hline \boldsymbol{\alpha}_{3} & 0 & \boldsymbol{\alpha}_{1} & 0 & \alpha_{3} & 0 & \alpha_{1} & 0 \\
\boldsymbol{\alpha}_{4} & 0 & 0 & \boldsymbol{\alpha}_{1} & \alpha_{4} & 0 & 0 & \alpha_{1} \\
0 & \boldsymbol{\alpha}_{4} & 0 & \boldsymbol{\alpha}_{2} & 0 & \alpha_{4} & 0 & \alpha_{2} \\
\hline \boldsymbol{\alpha}_{2} & \boldsymbol{\alpha}_{1} & 0 & 0 & \alpha_{2} & \alpha_{1} & 0 & 0 \\
0 & \boldsymbol{\alpha}_{3} & \boldsymbol{\alpha}_{2} & 0 & 0 & \alpha_{3} & \alpha_{2} & 0 \\
0 & 0 & \boldsymbol{\alpha}_{4} & \boldsymbol{\alpha}_{3} & 0 & 0 & \alpha_{4} & \alpha_{3} \\
2 \boldsymbol{\alpha}_{1} & 0 & 0 & 0 & 2 \alpha_{1} & 0 & 0 & 0 \\
0 & 2 \boldsymbol{\alpha}_{2} & 0 & 0 & 0 & 2 \alpha_{2} & 0 & 0 \\
0 & 0 & 2 \boldsymbol{\alpha}_{3} & 0 & 0 & 0 & 2 \alpha_{3} & 0 \\
0 & 0 & 0 & 2 \boldsymbol{\alpha}_{4} & 0 & 0 & 0 & 2 \alpha_{4}
\end{array}\right]
$$

ie

$$
\left[\begin{array}{cccc|cccc}
\alpha_{3} & 0 & 0 & 0 & 0 & 0 & \alpha_{1} & 0 \\
\alpha_{4} & 0 & 0 & 0 & 0 & 0 & 0 & \alpha_{1} \\
0 & \alpha_{4} & 0 & 0 & 0 & 0 & 0 & \alpha_{2} \\
\hline & {\left[D \Psi_{\widehat{P}}\right]} & & {\left[D \Psi_{\hat{P}}\right]}
\end{array}\right]
$$


see Section 4.3 for definition of $\left[D \Psi_{\hat{P}}\right]$. Here we note that

$$
\left[D \Psi_{\widehat{P}}\right]=\left[\begin{array}{cc}
D \Psi_{i j} & (i, j) \in E_{2, \widehat{P}} \\
D \Psi_{i j} & (i, j) \in E_{3, \widehat{P}} \\
D \Psi_{i i} & (i, i) \in E_{1, \widehat{P}}
\end{array}\right]=\left[\begin{array}{c}
D \Psi_{13} \\
D \Psi_{14} \\
D \Psi_{24} \\
D \Psi_{12} \\
D \Psi_{23} \\
D \Psi_{34} \\
D \Psi_{11} \\
D \Psi_{22} \\
D \Psi_{33} \\
D \Psi_{44}
\end{array}\right]=\left[\begin{array}{cccc}
\alpha_{3} & 0 & \alpha_{1} & 0 \\
\alpha_{4} & 0 & 0 & \alpha_{1} \\
0 & \alpha_{4} & 0 & \alpha_{2} \\
\alpha_{2} & \alpha_{1} & 0 & 0 \\
0 & \alpha_{3} & \alpha_{2} & 0 \\
0 & 0 & \alpha_{4} & \alpha_{3} \\
2 \alpha_{1} & 0 & 0 & 0 \\
0 & 2 \alpha_{2} & 0 & 0 \\
0 & 0 & 2 \alpha_{3} & 0 \\
0 & 0 & 0 & 2 \alpha_{4}
\end{array}\right] .
$$

Finally, using elementary column operations, we obtain

$$
\left[\begin{array}{c|c|c|c|cccc}
\alpha_{3} & 0 & -\alpha_{1} & 0 & 0 & 0 & \alpha_{1} & 0 \\
\alpha_{4} & 0 & 0 & -\alpha_{1} & 0 & 0 & 0 & \alpha_{1} \\
0 & \alpha_{4} & 0 & -\alpha_{2} & 0 & 0 & 0 & \alpha_{2} \\
\hline O_{10 \times 4} & O_{10 \times 4} & O_{10 \times 4} & O_{10 \times 4} & \multicolumn{3}{|c}{\left[D \Psi_{\hat{P}}\right]}
\end{array}\right]
$$

where $O_{s \times t}$ is the $s \times t$ zero matrix. Hence, the matrix is of $\operatorname{rank}=\operatorname{rank}\left[D \Psi_{\hat{P}}\right]+e_{2}$.

Now, we continue with the proof of Lemma 4.1. Using the notation as before, we recall our matrix $\left[D \Phi_{\hat{P}}\right]$ in (4-6). Now we use elementary row and column operations of $\left[D \Phi_{\hat{p}}\right]$ to obtain a matrix whose rank is easier to compute. The step will correspond to one after (4-7) in the above example.

First, for $(i, j) \in E_{2, \widehat{P}}$, add a row $\left[D \Phi_{i j}^{[1]}\right]$ of $\left[D \Phi_{\widehat{P}}\right]$ to another row $\left[D \Phi_{i j}^{[2]}\right]$ :

$$
\left(b_{[i]}^{[j]}, \alpha_{[j]}^{[i]}\right) \rightarrow\left(b_{[i]}^{[j]}+b_{[j]}^{[i]}, \alpha_{[i]}^{[j]}+\alpha_{[j]}^{[i]}\right) .
$$

Second, for $(i, j) \in E_{3, \hat{P}}$, multiply a row $\left[D \Phi_{i j}\right]$ of $\left[D \Phi_{\hat{P}}\right]$ by $a_{i j}^{-1}$ :

$$
\left(a_{i j} b_{[i]}^{[j]}+a_{j i} b_{[j]}^{[i]}, a_{j i} \alpha_{[i]}^{[j]}+a_{i j} \alpha_{[j]}^{[i]}\right) \rightarrow\left(b_{[i]}^{[j]}+b_{[j]}^{[i]}, \alpha_{[i]}^{[j]}+\alpha_{[j]}^{[i]}\right) .
$$

Recall that for $(i, j) \in E_{3, \widehat{P}}$ each $a_{i j}$ is nonzero and $a_{i j}=a_{j i}$ at the hyperbolic point in $\Phi^{-1}(0)$. 
Third, for $(i, i) \in E_{1, \widehat{P}}$, multiply a row $\left[D \Phi_{i i}\right]$ of $\left[D \Phi_{\widehat{P}}\right]$ by 2 :

$$
\left(b_{[i]}^{[i]}, \alpha_{[i]}^{[i]}\right) \rightarrow\left(2 b_{[i]}^{[i]}, 2 \alpha_{[i]}^{[i]}\right) .
$$

Fourth, multiply the left $(n+1) f$ columns of $\left[D \Phi_{\widehat{P}}\right]$ by 2 and the $(i(n+1)-n)^{\text {th }}$ columns $\left(i \in \mathbb{I}_{\hat{P}}\right)$ of $\left[D \Phi_{\widehat{P}}\right]$ by -1 respectively (The fact that

$$
\alpha_{i}=2 v_{i}^{t} J \quad \text { and } \quad b_{i}=v_{i}
$$

is used here):

$$
\begin{array}{ll}
{\left[D \Phi_{i j}^{[1]}\right] \rightarrow\left(\alpha_{[j]}^{[i]}, \alpha_{[i]}^{[j]}\right)} & (i, j) \in E_{2, \widehat{P}}, \\
{\left[D \Phi_{i j}^{[2]}\right] \rightarrow\left(\alpha_{[i]}^{[j]}+\alpha_{[j]}^{[i]}, \alpha_{[i]}^{[j]}+\alpha_{[j]}^{[i]}\right)} & (i, j) \in E_{2, \widehat{P},}, \\
{\left[D \Phi_{i j}\right] \rightarrow\left(\alpha_{[i]}^{[j]}+\alpha_{[j]}^{[i]}, \alpha_{[i]}^{[j]}+\alpha_{[j]}^{[i]}\right)} & (i, j) \in E_{3, \widehat{P}}, \\
{\left[D \Phi_{i i}\right] \rightarrow\left(2 \alpha_{[i]}^{[i]}, 2 \alpha_{[i]}^{[i]}\right)} & (i, i) \in E_{1, \widehat{P}} .
\end{array}
$$

Similarly, the rows of the $(f+e) \times(n+1) f$ Jacobian matrix $\left[D \Psi_{\hat{P}}\right]$ are as follows:

$$
\begin{array}{ll}
{\left[D \Psi_{i j}\right]=\alpha_{[i]}^{[j]}+\alpha_{[j]}^{[i]},} & (i, j) \in E_{2, \widehat{P}} \cup E_{3, \widehat{P}}, \\
{\left[D \Psi_{i i}\right]=2 \alpha_{[i]}^{[i]},} & (i, i) \in E_{1, \widehat{P}}
\end{array}
$$

see Section 4.3 for definition of $\left[D \Psi_{\widehat{P}}\right]$. Comparing these two matrices and rearranging, we observe that $\left[D \Phi_{\hat{P}}\right]$ became

$$
\left[\begin{array}{c|c}
\alpha_{\left[\mathbb{P}_{\hat{P}}(1,1)\right]}^{[1]} & \alpha_{[1]}^{\left[\mathbb{I}_{\hat{P}}(1,1)\right]} \\
\vdots & \vdots \\
\alpha_{\left[\mathbb{I}_{\hat{P}}(1, i(1))\right]}^{[1]} & \alpha_{[1]}^{\left[\mathbb{I}_{\hat{P}}(1, i(1))\right]} \\
\vdots & \vdots \\
\alpha_{\left[\mathbb{I}_{\hat{P}}(q, 1)\right]}^{[q]} & \alpha_{[q]}^{\left[\mathbb{I}_{\hat{P}}(q, 1)\right]} \\
\vdots & \vdots \\
\alpha_{\left[\mathbb{I}_{\hat{P}}(q, i(q))\right]}^{[q]} & \alpha_{[q]}^{\left[\mathbb{I}_{\hat{P}}(q, i(q))\right]} \\
\hline\left[D \Psi_{\hat{P}}\right] & {\left[D \Psi_{\hat{P}}\right]}
\end{array}\right]
$$

which is an $N_{\widehat{P}} \times 2(n+1) f$ matrix divided into two $e_{2} \times(n+1) f$-matrices. The top ones correspond to the copies of $E_{2, \widehat{P}^{-}}$-rows, and two bottom $(f+e) \times(n+1) f-$ matrices equal $\left[D \Psi_{\hat{P}}\right]$. 
Using elementary column operations, we obtain

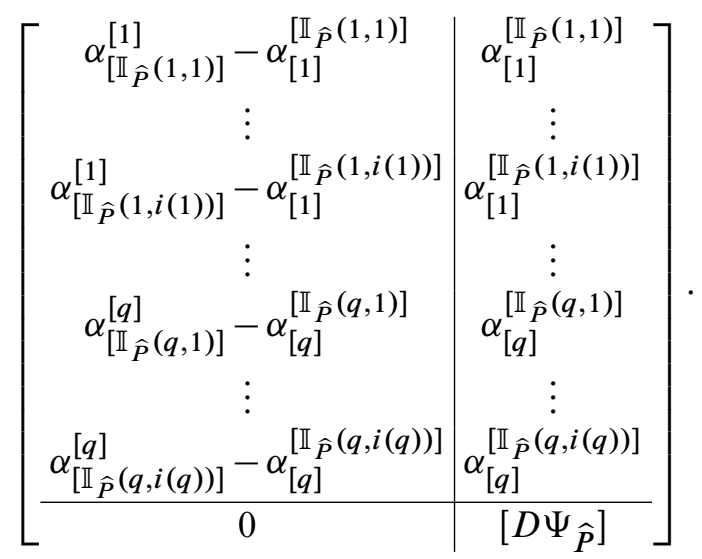

Now rewriting this matrix into the union of $f$ of $N_{\widehat{P}} \times(n+1)$-matrices and an $N_{\widehat{P}} \times f(n+1)$ matrix, we obtain

$\left[\begin{array}{c|c|c|c|c|c}\alpha_{\mathbb{I}_{\widehat{P}}(1,1)} & * & * & * & \cdots & * \\ \vdots & \vdots & \vdots & \vdots & \ddots & \vdots \\ \alpha_{\mathbb{I}_{\hat{P}}(1, i(1))} & * & * & * & \cdots & * \\ \hline 0 & \alpha_{\mathbb{I}_{\hat{P}}(2,1)} & * & * & \cdots & * \\ \vdots & \vdots & \vdots & \vdots & \ddots & \vdots \\ 0 & \alpha_{\mathbb{I}_{\widehat{P}}(2, i(2))} & * & * & \cdots & * \\ \hline \vdots & \vdots & \ddots & \vdots & \ddots & \vdots \\ \hline 0 & 0 & \cdots & \alpha_{\mathbb{I}_{\widehat{P}}(q, 1)} & \cdots & * \\ \vdots & \vdots & \vdots & \vdots & \ddots & \vdots \\ 0 & 0 & \cdots & \alpha_{\mathbb{I}_{\widehat{P}}(q, i(q))} & \cdots & * \\ \hline 0 & 0 & \cdots & 0 & 0 & {\left[D \Psi_{\widehat{P}}\right]}\end{array}\right]$

where 0 's are zero matrices. The matrix is so that

$$
\left[\begin{array}{c}
\alpha_{\mathbb{I}_{\widehat{P}}(k, 1)} \\
\alpha_{\mathbb{I}_{\widehat{P}}(k, 2)} \\
\vdots \\
\alpha_{\mathbb{I}_{\widehat{P}}(k, i(k))}
\end{array}\right]
$$

is in the $k^{\text {th }}$ column from rows $\sum_{j=1}^{k-1} i(j)+1$ to $\sum_{j=1}^{k} i(j)$ and every entry below is zero for $k=1, \ldots, q$. 
The general position condition of the weak orderability implies that for each $k \in$ $\{1,2, \ldots, q\}$,

$$
\alpha_{\mathbb{I}_{\hat{P}}(k, 1)}, \quad \alpha_{\mathbb{I}_{\hat{P}}(k, 2)}, \quad \cdots, \quad \alpha_{\mathbb{I}_{\hat{P}}(k, i(k))}
$$

are linearly independent, ie all submatrices

$$
\left[\begin{array}{c}
\alpha_{\mathbb{I}_{\widehat{P}}(k, 1)} \\
\alpha_{\mathbb{I}_{\widehat{P}}(k, 2)} \\
\vdots \\
\alpha_{\mathbb{I}_{\widehat{P}}(k, i(k))}
\end{array}\right]
$$

are of full rank. This establishes the result.

\subsection{Proof of Theorem 4.1}

Let $\widehat{P}$ be a compact Coxeter $n$-orbifold admitting a hyperbolic structure. Assume that $\widehat{P}$ admits a real projective structure, but does not admit a spherical or Euclidean structure. Define

$$
\begin{aligned}
\mathcal{V}_{\widehat{P}} & :=\left\{p \in \mathcal{U}_{\widehat{P}} \subset\left(V^{*}\right)^{f} \times V^{f} \mid D_{p} \Phi_{\widehat{P}} \text { is surjective }\right\}, \\
\tilde{\mathbb{D}}(\widehat{P})_{r} & :=\tilde{\mathbb{D}}(\widehat{P}) \cap \mathcal{V}_{\widehat{P}}=\Phi_{\widehat{P}}^{-1}(0) \cap \mathcal{V}_{\widehat{P}} .
\end{aligned}
$$

The second one is an open subset since the maximal rank condition is an open condition since the rank condition expresses the independence of the row vectors of the differential. Since $\Phi_{\widehat{P}}$ is $\widetilde{G}$-invariant, $\widetilde{G}$ acts on $\tilde{\mathbb{D}}(\widehat{P})_{r}$. The action of $\widetilde{G}$ on $\widetilde{\mathbb{D}}(\widehat{P})_{r}$ is induced from the action $\theta$ on $\widetilde{\mathbb{D}}(\widehat{P})$ in (3-2). Recall that $N_{\widehat{P}}=f+e+e_{2}$, where $f$, e and $e_{2}$ are the number of facets, ridges and ridges of order 2 of $\widehat{P}$ respectively.

We use the following steps:

(1) $\tilde{\mathbb{D}}(\widehat{P})_{r}$ is a smooth manifold of dimension $2(n+1) f-N_{\widehat{P}}$ if $\widetilde{\mathbb{D}}(\widehat{P})_{r} \neq \varnothing$.

(2) The orbit space $\mathbb{D}(\widehat{P})_{r}:=\widetilde{\mathbb{D}}(\widehat{P})_{r} / \widetilde{G}$ is a smooth manifold of dimension $\operatorname{dim} \tilde{\mathbb{D}}(\widehat{P})_{r}-\operatorname{dim} \widetilde{G}$, and it identifies with an open subset of $\mathbb{D}(\widehat{P})$.

(3) Moreover, if $P$ satisfies the condition $(\mathrm{C} 1)$, then the manifold $\mathbb{D}(\widehat{P})_{r}$ is of dimension $e_{+}(\widehat{P})-n$.

(4) Furthermore, if $\widehat{P}$ admits a hyperbolic structure and satisfies the condition (C2), the hyperbolic point $t$ is in $\mathbb{D}(\widehat{P})_{r}$. This will complete the proof of Theorem 4.1.

We now prove them one by one. 
(1) The set $\mathcal{V}_{\widehat{P}}$ is an open subset of $\mathcal{U}_{\widehat{P}} \subset\left(V^{*}\right)^{f} \times V^{f}$ and the restriction to $\mathcal{V}_{\widehat{P}}$ of the map $\Phi_{\widehat{P}}$ is a submersion. Thus each level set of $\left.\Phi_{\widehat{P}}\right|_{\mathcal{V}_{\hat{P}}}$ is an embedded submanifold in $\mathcal{V}_{\widehat{P}}$ whose codimension is $N_{\widehat{P}}$. The conclusion is immediate.

(2) As we defined above, $\tilde{\mathbb{D}}(\widehat{P})_{r}$ is an open subset of $\tilde{\mathbb{D}}(\widehat{P})$ that is the complement of an algebraic closed set and $\widetilde{G}$ acts on both sets. By Lemma 4.2, $\widetilde{G}$ acts smoothly, freely and properly on a smooth manifold $\widetilde{\mathbb{D}}(\widehat{P})_{r}$, and hence the orbit space $\widetilde{\mathbb{D}}(\widehat{P})_{r} / \widetilde{G}$ is a smooth manifold of dimension $\operatorname{dim} \widetilde{\mathbb{D}}(\widehat{P})_{r}-\operatorname{dim} \widetilde{G}$. Therefore, $\widetilde{\mathbb{D}}(\widehat{P})_{r} / \widetilde{G}$ identifies with an open subset of $\mathbb{D}(\widehat{P})$ by Theorem 3.2.

(3) The equation $\operatorname{dim} \widetilde{\mathbb{D}}(\widehat{P})_{r}-\operatorname{dim} \widetilde{G}=\left(2(n+1) f-N_{\widehat{P}}\right)-\left(f+(n+1)^{2}-1\right)=$ $e_{+}-n-2 \delta_{P}$ holds. Since $\delta_{P}=0$, we obtain $\operatorname{dim} \widetilde{\mathbb{D}}(\hat{P})_{r}-\operatorname{dim} \widetilde{G}=e_{+}(\hat{P})-n$, and hence the step (2) implies the conclusion.

(4) Proposition 4.1 yields $\operatorname{ker} D \Psi_{\widehat{P}}=\frac{1}{2} n(n+1)$ at the hyperbolic point $t$. Hence

$$
\operatorname{rank} D \Psi_{\widehat{P}}=(n+1) f-\frac{1}{2} n(n+1)=f+e-\delta_{P}
$$

holds where $\delta_{P}=e-n f+\frac{1}{2} n(n+1)$. Since $\delta_{P}=0$ and $\hat{P}$ is weakly orderable, $\operatorname{rank} D \Phi_{\widehat{P}}=\operatorname{rank} D \Psi_{\widehat{P}}+e_{2}=f+e+e_{2}$ at $t$ by Lemma 4.1, and so $D \Phi_{\widehat{P}}$ at $t$ is of full rank.

Lemma 4.2 Let $\widehat{P}$ be a compact Coxeter $n$-orbifold. Assume that $\widehat{P}$ admits a real projective structure, but does not admit a spherical or Euclidean structure. Then $\widetilde{G}$ acts smoothly, freely and properly on a smooth manifold $\widetilde{\mathbb{D}}(\widehat{P})_{r}$.

Proof We show that $\widetilde{G}$ acts freely on a smooth manifold $\widetilde{\mathbb{D}}(\widehat{P})_{r}$, a locally compact metric space. Suppose that

$$
\left(d_{1}, \ldots, d_{f}, g\right) \cdot\left(\alpha_{1}, \ldots, \alpha_{f}, b_{1}, \ldots, b_{f}\right)=\left(\alpha_{1}, \ldots, \alpha_{f}, b_{1}, \ldots, b_{f}\right),
$$

where $d_{1}, \ldots, d_{f} \in \mathbb{R}_{+}$and $g \in \mathrm{SL}_{n+1}^{ \pm}(\mathbb{R})$. That is,

$$
d_{i} \alpha_{i} g^{-1}=\alpha_{i} \quad \text { and } \quad d_{i}^{-1} g b_{i}=b_{i} \quad \text { for every } i \in \mathbb{I} .
$$

Hence, $d_{i} d_{j}^{-1} a_{i j}=a_{i j}$ holds, and $d_{i}=d_{j}$ if $\alpha_{i}\left(b_{j}\right) \neq 0$.

By Proposition 2.3, for any holonomy group $\Gamma$ of $\pi_{1}(\hat{P})$, the Cartan matrix of $\Gamma$ is indecomposable. It follows that $d_{1}=\cdots=d_{f}$. Denote the common value by $d$. Choose $(n+1)$ linearly independent linear functionals $\alpha_{i_{0}}, \alpha_{i_{1}}, \ldots, \alpha_{i_{n}}$ from the facets of $\widehat{P}$ since the fundamental domain is a properly convex polytope by the condition 
of $\mathcal{U}_{\widehat{\boldsymbol{P}}}$. Let $S$ be an invertible $(n+1) \times(n+1)$ matrix

$$
S=\left[\begin{array}{c}
\alpha_{i_{0}} \\
\alpha_{i_{1}} \\
\vdots \\
\alpha_{i_{n}}
\end{array}\right]
$$

Then $d S g^{-1}=S$ and hence $d^{n+1}=\operatorname{det}(g)=1$. Observe that $d=1$ and $g=I_{n+1}$ establishing the result.

Next, we show that $\widetilde{G}$ acts properly on a smooth manifold $\widetilde{\mathbb{D}}(\widehat{P})_{r}$. Suppose that a sequence $\left\{p_{k}=\left(\alpha_{1, k}, \ldots, \alpha_{f, k}, b_{1, k}, \ldots, b_{f, k}\right)\right\}$ in $\widetilde{G}$ is such that

$$
\left\{p_{k}\right\} \rightarrow\left(\alpha_{1}, \ldots, \alpha_{f}, b_{1}, \ldots, b_{f}\right) \in \tilde{\mathbb{D}}(\widehat{P})_{r},
$$

and $\left\{q_{k}=\left(d_{1, k}, \ldots, d_{f, k}, g_{k}\right)\right\}$ is a sequence in $\widetilde{G}$ such that

$$
q_{k} \cdot p_{k} \rightarrow\left(\tilde{\alpha}_{1}, \ldots, \tilde{\alpha}_{f}, \tilde{b}_{1}, \ldots, \tilde{b}_{f}\right) \in \tilde{\mathbb{D}}(\hat{P})_{r} \quad \text { as } k \rightarrow \infty .
$$

That is,

$$
\left\{d_{i, k} \alpha_{i, k} g_{k}^{-1}\right\} \rightarrow \widetilde{\alpha}_{i} \quad \text { and } \quad\left\{d_{i, k}^{-1} g_{k} b_{i, k}\right\} \rightarrow \widetilde{b}_{i} \quad \text { for each } i \in \mathbb{I} .
$$

Since we are in a metric space, we show that $\left\{q_{k}\right\}$ is bounded to prove the properness of the action: We have

$$
\left\{d_{i, k} d_{j, k}^{-1} \alpha_{i, k} b_{j, k}\right\} \rightarrow \widetilde{\alpha}_{i} \tilde{b}_{j}
$$

hence

$$
\left\{d_{i, k} d_{j, k}^{-1}\right\} \rightarrow \tilde{\alpha}_{i} \tilde{b}_{j}\left(\alpha_{i} b_{j}\right)^{-1} \quad \text { if } \alpha_{i} b_{j} \neq 0
$$

Moreover,

$$
\widetilde{\alpha}_{i} \tilde{b}_{j}, \widetilde{\alpha}_{j} \tilde{b}_{i}, \alpha_{i} b_{j}, \alpha_{j} b_{i}<0, \quad(i, j) \notin E_{1} \cup E_{2} .
$$

Since the Cartan matrices $A=\left(a_{i j}\right), a_{i j}=\alpha_{i} b_{j}$ and $\tilde{A}=\left(\tilde{a}_{i j}\right), \tilde{a}_{i j}=\tilde{\alpha}_{i} \tilde{b}_{j}$, are indecomposable,

$$
\left\{d_{i, k} d_{j, k}^{-1}\right\} \rightarrow c_{i j}>0 \quad \text { for every }(i, j) \in \mathbb{I} \times \mathbb{I} .
$$

Define $(n+1) \times(n+1)$ matrices

$$
\widetilde{S}=\left[\begin{array}{c}
\tilde{\alpha}_{i_{0}} \\
\tilde{\alpha}_{i_{1}} \\
\vdots \\
\tilde{\alpha}_{i_{n}}
\end{array}\right], \quad S=\left[\begin{array}{c}
c_{i_{0} i_{0}} \alpha_{i_{0}} \\
c_{i_{1} i_{0}} \alpha_{i_{1}} \\
\vdots \\
c_{i_{n} i_{0}} \alpha_{i_{n}}
\end{array}\right] \quad \text { and } \quad S_{k}=\left[\begin{array}{c}
d_{i_{0}, k} \alpha_{i_{0}, k} \\
d_{i_{1}, k} \alpha_{i_{1}, k} \\
\vdots \\
d_{i_{n}, k} \alpha_{i_{n}, k}
\end{array}\right] \text {. }
$$


Since $\tilde{S}$ and $S$ are invertible, (4-9) and (4-10) show that

$$
\left\{S_{k} g_{k}^{-1}\right\} \rightarrow \widetilde{S} \quad \text { and } \quad\left\{d_{i_{0}, k}^{-1} S_{k}\right\} \rightarrow S
$$

hence

$$
\left\{d_{i_{0}, k} g_{k}^{-1}\right\} \rightarrow S^{-1} \widetilde{S} .
$$

As det $g_{k}= \pm 1$, the sequence $\left\{d_{i_{0}, k}^{n+1}\right\}$ converges to a positive number

$$
\left|\operatorname{det}\left(S^{-1} \tilde{S}\right)\right| \text {. }
$$

Denote by $d_{i_{0}}$ the positive $(n+1)^{\text {th }}$ root of this limit. $\left\{d_{i_{0}, k}\right\} \rightarrow d_{i_{0}}$ and $\left\{g_{k}\right\}$ limits to $\left\{d_{i_{0}} \widetilde{S}^{-1} S\right\}$ respectively. Since we can choose a collection of faces to include $\alpha_{i_{0}}$ for any $i_{0},\left\{q_{k}\right\}$ is convergent.

\subsection{Proofs of Corollaries $\mathbf{1 . 1}$ and $\mathbf{1 . 2}$}

Proof of Corollary 1.1 We have that $\delta_{P}=0$ for any 3-dimensional simple polytope $P$ by Euler's formula. Hence, Theorem 4.1 gives us the conclusion.

Proof of Corollary 1.2 Let $\widehat{P}$ be a hyperbolic truncation Coxeter orbifold with the fundamental polytope $P \subset \mathbb{S}^{n}$. For $n=3$, this is the work of Marquis [40]. For $n \geq 4$, as shown in Brøndsted [11, Section 19], $P$ is a truncation $n$-polytope if and only if $\delta_{P}=0$.

By Lemma 4.3, an orbifold based on a truncation $n$-polytope $P$ is weakly orderable since a compact Coxeter orbifold based on an $n$-simplex is weakly orderable.

Lemma 4.3 Let a polytope $P_{2}$ be obtained from a polytope $P_{1}$ by iterated truncation. Suppose that a compact Coxeter orbifold $\hat{P}_{2}$ has the base polytope $P_{2}$ and another compact Coxeter orbifold $\hat{P}_{1}$ has the base polytope $P_{1}, \hat{P}_{2}$ has the ridge orders extending those of $\widehat{P}_{1}$, and $\widehat{P}_{1}$ is weakly orderable. Then $\widehat{P}_{2}$ is weakly orderable.

Proof By induction, suppose that $P_{2}$ obtained from $P_{1}$ by a truncation at a vertex $v$ of $P_{1}$. We give an ordering of faces of $P_{2}$ by labeling the new facet to be the lowest one $F_{1}$ and the remaining ones are to be denoted $F_{i+1}$ when they were labeled by $F_{i}$ before. Then we need to check for $F_{1}$ only since the other faces already satisfy the weak orderability condition for those faces. However, $F_{1}$ meets only $n$ facets by the simplicity of $P_{1}$ and since these $n$ facets were meeting at a vertex only, $F_{1}$ can only be an $(n-1)$-dimensional simplex. This implies that any collection of the facets meeting $F_{1}$ are in a general position. 


\section{Examples}

Section 5 provides several examples of weakly orderable compact hyperbolic Coxeter 3 -orbifolds and gives two examples satisfying only one of the two conditions (C1) and (C2) where the conclusion of Theorem 4.1 does not hold.

\subsection{Weakly orderable compact hyperbolic Coxeter 3-orbifolds}

Every compact hyperbolic Coxeter 3-orbifold whose base polytope has the combinatorial type of a cube is weakly orderable. Theorem 1.1 shows that almost all compact hyperbolic Coxeter 3-orbifolds, with the combinatorial type of a dodecahedron, are weakly orderable while there are ones not weakly orderable.

Before going to the proof of Theorem 1.1, we state Tutte's theorem [46].

A 1-dimensional cell complex $\mathbb{G}$ is a graph. It consists of vertices (0-cells) to which edges (1-cells) are attached. We deal with only simple graphs, that have no loops and no more than one edge between any two vertices. The degree of a vertex in a graph is the number of edges with which it is incident. If all the vertices in a graph $\mathbb{G}$ have degree $d, \mathbb{G}$ is said to be regular of degree $d$.

A subgraph of $\mathbb{G}$ is a graph having all of its vertices and edges in $\mathbb{G}$. A graph $\mathbb{G}$ with at least $k+1$ vertices is $k$-connected if every subgraph of $\mathbb{G}$, obtained by omitting from $\mathbb{G}$ any $k-1$ or fewer vertices and the edges incident to them, is connected. A spanning subgraph of $\mathbb{G}$ is a subgraph containing all the vertices of $\mathbb{G}$. A factor is a spanning subgraph which is regular of degree 1.

Theorem 5.1 (Tutte [46]) Let $\mathbb{G}$ be a finite graph. If $\mathbb{G}$ is a $d$-connected graph having the even number of vertices and is regular of degree $d$, then $\mathbb{G}$ has a factor. Moreover, if in addition $\mathfrak{e}$ is any edge of $\mathbb{G}$, then $\mathbb{G}$ has a factor containing $\mathfrak{e}$.

Lemma 5.1 Let $P$ be a properly convex compact 3-polytope but not a tetrahedron. Suppose that $P$ has no prismatic 3-circuit and has at most one prismatic 4-circuit. Then there exists a compact hyperbolic Coxeter 3 -orbifold $\widehat{P}$ with the base polytope $P$ such that each vertex is incident with exactly two edges of order 2.

Proof Assume that four 2-cells $F_{i}, F_{j}, F_{k}$ and $F_{l}$ of $P$ form a prismatic 4-circuit. Denote the edge $F_{i} \cap F_{j}$ by $\mathfrak{e}$. By Steinitz's theorem, the graph $\mathbb{G}=\mathbb{G}(P)$ of $P$ is 3-connected; see Grünbaum [33, Chapter 13]. Since $P$ is simple, $\mathbb{G}$ is regular of degree 3 and the number of vertices is even. By Tutte's theorem, $\mathbb{G}$ has a factor $\mathbb{F}$ containing $\mathfrak{e}$. If $P$ has no prismatic 4 -circuit, then we choose an arbitrary factor $\mathbb{F}$ 
of $\mathbb{G}$. Every vertex of $P$ is incident with two edges in $\mathbb{G} \backslash \mathbb{F}$ and one edge in $\mathbb{F}$. Observe that

$$
\frac{\pi}{k}+\frac{\pi}{2}+\frac{\pi}{2}>\pi \quad \text { and } \quad \frac{\pi}{k}+\frac{\pi}{2}+\frac{\pi}{2}+\frac{\pi}{2}<2 \pi \quad \text { for every integer } k \geq 3 .
$$

Andreev's theorem (see Roeder, Hubbard and Dunbar [43, Theorem 1.4 and Proposition 1.5]) yields a compact hyperbolic Coxeter 3 -orbifold $\hat{P}$ such that every edge in $\mathbb{G} \backslash \mathbb{F}$ (resp. $\mathbb{F}$ ) is of order 2 (resp. of order $k \neq 2$ ), corresponding to a dihedral angle $\pi / 2$ (resp. $\pi / k)$.

Let $\mathbb{G}$ be a finite graph, and let $L$ be a set. Denote by $E(\mathbb{G})$ the set of edges of $\mathbb{G}$. A function $\vartheta: E(\mathbb{G}) \rightarrow L$ is called an edge-labeling function, and we call a pair $(\mathbb{G}, \vartheta)$ an edge-labeled graph. An edge $\mathfrak{e}$ is called an $l$-edge if $\vartheta(\mathfrak{e})=l$.

In this section, we consider the edge-labeled graph $(\mathbb{G}, \vartheta)$ satisfying the following conditions.

(E1) $\mathbb{G}$ is simple, planar and 3-connected.

(E2) $\mathbb{G}$ is regular of degree 3.

(E3) The set $L$ of labels is $\{0,1\}$.

(E4) Every vertex of $\mathbb{G}$ is incident with three edges $\mathfrak{e}_{1}, \mathfrak{e}_{2}$ and $\mathfrak{e}_{3}$ such that

$$
\vartheta\left(\mathfrak{e}_{1}\right)+\vartheta\left(\mathfrak{e}_{2}\right)+\vartheta\left(\mathfrak{e}_{3}\right) \equiv 1(\bmod 2) .
$$

If $(\mathbb{G}, \vartheta)$ can be ordered so that each face contains at most three 0 -edges in faces of higher indices, $(\mathbb{G}, \vartheta)$ is said to be weakly orderable. (Here (E1) holds if and only if $\mathbb{G}$ is isomorphic to the 1-skeleton of a properly convex 3-polytope by Steinitz's theorem.)

Let $P$ be a properly convex 3 -polytope, and let $\mathbb{G}$ be the 1 -skeleton of $P$ as an abstract 3-polyhedron. The graph $\mathbb{G}$ is embedded in the 2-dimensional sphere $S^{2}$ homeomorphic to the boundary of $P$. We call a face of $P$ a face of $\mathbb{G}$. The respective numbers of vertices, edges and faces of $\mathbb{G}$ shall be denoted by $v, e$ and $f$.

Lemma 5.2 Let $(\mathbb{G}, \vartheta)$ be an edge-labeled graph satisfying the conditions (E1)-(E4). Then the number of 0 -edges of at least one face $F$ of $\mathbb{G}$ is less than or equal to 3.

Proof Denote by $e_{2}$ the number of 0 -edges. Condition (E1) implies that $v-e+f=2$, (E2) implies that $2 e=3 v$, and (E3) and (E4) imply that $2 e_{2} \leq 2 v$. By an elementary computation, we obtain $2 e_{2} \leq 4(f-2)<4 f$. The conclusion is immediate. 
We define an edge-deletion for an edge-labeled graph satisfying conditions (E1)-(E4). When each pair of edges ending at a vertex $\mathfrak{a}$ or $\mathfrak{b}$ of an edge $\mathfrak{e}$ in $(\mathbb{G}, \vartheta)$ have the same labels, we can delete $\mathfrak{e}$ from $(\mathbb{G}, \vartheta)$ and amalgamate the pair of edges incident to $\mathfrak{a}$ and the pair for $\mathfrak{b}$ (see Figure 2). We define the edge-deletion on $\mathbb{G}$ satisfying $(\mathrm{E} 1)$ and (E2) similarly. Edge-deletion preserves conditions (E2)-(E4) for $(\mathbb{G}, \vartheta)$ (just $(\mathrm{E} 2)$ for $\mathbb{G}$ ).

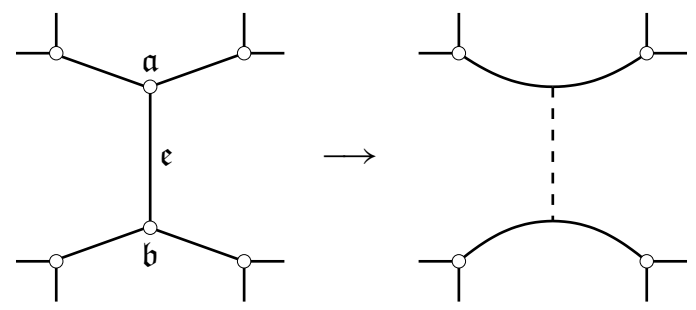

Figure 2: An edge-deleting operation

Let $\mathbb{G}$ be a graph satisfying conditions (E1)-(E2). An edge $\mathfrak{e}$ of $\mathbb{G}$ is said to be removable when the graph obtained from $\mathbb{G}$ by deleting the edge $\mathfrak{e}$ remains to satisfy the condition (E1) (and (E2) obviously).

Theorem 5.2 (Fouquet [28, Corollary 2.7]) Let $\mathbb{G}$ be a graph with more than 6 edges satisfying conditions (E1)-(E2), and let $C$ be a cycle of $\mathbb{G}$. Then $C$ contains at least two removable edges.

Lemma 5.3 Let $P$ be a properly convex compact 3 -polytope, and let $\widehat{P}$ be the Coxeter 3-orbifold arising from $P$. Assume that every vertex of $\widehat{P}$ is incident with two edges of order 2 and one edge of order greater than or equal to 3 . Then $\hat{P}$ is weakly orderable.

Proof Let $\widehat{\mathbb{G}}$ be the graph of the 3 -polytope $P$. Define the edge-labeling function $\widehat{\vartheta}$ by

$$
\hat{\vartheta}(\mathfrak{e})= \begin{cases}0 & \text { if the edge } \mathfrak{e} \text { is of order } 2, \\ 1 & \text { otherwise }\end{cases}
$$

Then the edge-labeled graph $(\widehat{\mathbb{G}}, \widehat{\vartheta})$ satisfies conditions (E1)-(E4) by Steinitz's theorem.

Given a labeled graph $(\mathbb{G}, \vartheta)$ satisfying (E1)-(E4) and a face $F$, we can reverse the label for every edge of $F$ and the new labeling function on $\mathbb{G}$ will still satisfy (E1)-(E4). 
We show that if $(\mathbb{G}, \vartheta)$ satisfies conditions $(\mathrm{E} 1)-(\mathrm{E} 4)$, then $(\mathbb{G}, \vartheta)$ is weakly orderable. The proof proceeds by induction on the number $f$ of faces of $\mathbb{G}$. The condition (E1) implies $f \geq 4$. We have $f=4$ if and only if $\mathbb{G}$ is the graph of a tetrahedron. In this case $\mathbb{G}$ is weakly orderable.

Now assume that $\mathbb{G}$ has $f$ faces for $f \geq 5$ and that any labeled graph $\left(\mathbb{G}^{\prime}, \vartheta^{\prime}\right)$ satisfying (E1)-(E4) is weakly orderable provided that the number of faces is less than $f$. By Lemma 5.2, $\mathbb{G}$ has a face $F$ such that the number of 0 -edges of $F$ is less than or equal to 3 as $e=3(f-2)$ by Euler's formula. By Theorem 5.2, the cycle $\partial F$ contains a removable edge $\mathfrak{e}$.

- If we have $\vartheta(\mathfrak{e})=1$, then each pair of edges which are adjacent to a vertex of $\mathfrak{e}$ have the same label. Then let $\vartheta^{\prime}:=\vartheta$.

- Otherwise, $\vartheta(\mathfrak{e})=0$. We relabel every edge in the cycle $\partial F$ to become the edge of the opposite label, and obtain the new label function $\vartheta^{\prime}$ of $\mathbb{G}$ such that $\vartheta^{\prime}(\mathfrak{e})=1$. The resulting edge-labeled graph $\left(\mathbb{G}, \vartheta^{\prime}\right)$ still satisfies the conditions (E1)-(E4). Also each pair of edges which are adjacent to a vertex of $\mathfrak{e}$ have the same label.

Denote by $F^{\prime}$ the face adjacent to $F$ such that $F \cap F^{\prime}=\mathfrak{e}$. We can delete the edge $\mathfrak{e}$ of $\left(\mathbb{G}, \vartheta^{\prime}\right.$ ). Two adjacent faces $F$ and $F^{\prime}$ are amalgamated into a face $F^{\prime \prime}$ (see Figure 3 ).

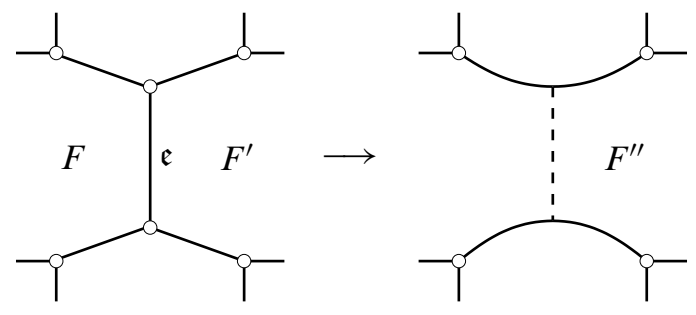

Figure 3: Amalgamating two adjacent facets into a facet

Now, the resulting edge-labeled graph $(\widetilde{\mathbb{G}}, \widetilde{\vartheta})$ has fewer faces but still satisfies all conditions (E1)-(E4) since $\mathfrak{e}$ is removable. Using the induction hypothesis, the edgelabeled graph $(\widetilde{\mathbb{G}}, \widetilde{\vartheta})$ is weakly orderable, and hence we label the faces of $\widetilde{G}$ with the indices $\{2,3, \ldots, f\}$. Now we reinsert $\mathfrak{e}$ and recover the old labels $\vartheta$ of $\mathbb{G}$ by

- doing nothing, or

- reversing the labels of the edges of $F$ provided that we reversed the labels of edges of $F$ above. 
Let $F$ be the first face of $(\mathbb{G}, \vartheta)$, and we label all the other faces of $(\mathbb{G}, \vartheta)$ by inheriting the ordering of faces of $(\widetilde{\mathbb{G}}, \widetilde{\vartheta})$. Since the number of 0 -edges of $F$ under $\vartheta$ is less than or equal to $3,(\mathbb{G}, \vartheta)$ is weakly orderable with the indices $\{1,2, \ldots, f\}$.

Proof of Theorem 1.1 By Lemma 5.1, there exists a compact hyperbolic Coxeter orbifold $\hat{P}$ whose base polytope is combinatorially equivalent to $P$. Let $e$ be the number of edges of $P$, and let $p=\frac{1}{3} e$. Observe that $p \in \mathbb{Z}_{+}$by the vertex incidence condition. Let $\mathcal{N}(d)$ be the set of compact hyperbolic Coxeter orbifolds whose base polytopes are combinatorially equivalent to $P$ and whose edge orders are less than or equal to $d$. For each integer $d \geq 7$ and $j \in\{0,1, \ldots, e\}$, we define

$\mathcal{N}_{\omega}(d)=\{\widehat{P} \in \mathcal{N}(d) \mid \widehat{P}$ is weakly orderable $\}$,

$\mathcal{N}_{j}(d)=\{\widehat{P} \in \mathcal{N}(d) \mid$ the number of edges of order greater than or equal to 7 in $\widehat{P}\}$.

Assume that $\widehat{P}$ is a compact hyperbolic Coxeter 3-orbifold. By the orbifold condition, if an edge $\mathfrak{e}$ of $\widehat{P}$ is of order $\geq 7$, then edges which are adjacent to $\mathfrak{e}$ are of order 2 . Therefore the number of edges of order $\geq 7$ in $\widehat{P}$ is less than or equal to $p=\frac{1}{3} e$. In other words, $\mathcal{N}_{j}(d)=\varnothing$ for every $j>p$.

We have

$$
|\mathcal{N}(d)|=\sum_{j=0}^{p}\left|\mathcal{N}_{j}(d)\right| .
$$

Moreover, observe that $\widehat{P} \in \mathcal{N}_{p}(d)$ if and only if every vertex of $\widehat{P}$ is incident with two edges of order 2 and one edge of order greater than or equal to 7 . For any fixed integers $l, m \geq 2$,

$\frac{1}{k}+\frac{1}{l}+\frac{1}{m}>1$ for some integer $k \geq 7 \Leftrightarrow \frac{1}{k}+\frac{1}{l}+\frac{1}{m}>1$ for each integer $k \geq 7$.

Consequently for each $d \in\{7,8, \ldots\}$ we have

$$
\left|\mathcal{N}_{j}(d)\right|=\left|\mathcal{N}_{j}(7)\right| \cdot(d-6)^{j} .
$$

Lemma 5.1 shows that $\mathcal{N}_{p}(7) \neq \varnothing$ and Lemma 5.3 implies that

$$
\frac{\left|\mathcal{N}_{\omega}(d)\right|}{|\mathcal{N}(d)|} \geq \frac{\left|\mathcal{N}_{p}(d)\right|}{|\mathcal{N}(d)|}=\frac{\left|\mathcal{N}_{p}(7)\right| \cdot(d-6)^{p}}{\sum_{j=0}^{p}\left|\mathcal{N}_{j}(7)\right| \cdot(d-6)^{j}}
$$

establishing the result.

Example 5.1 Let $m$ be an integer greater than or equal to 5. A Löbell 3-polytope $L(m)$ is a 3 -polytope with $(2 m+2)$ faces where upper and lower sides are $m$-gons, and the complementary surface is a union of $2 m$ pentagons, arranged similarly as in the dodecahedron. Figure 4 shows the case when $m=6$. 


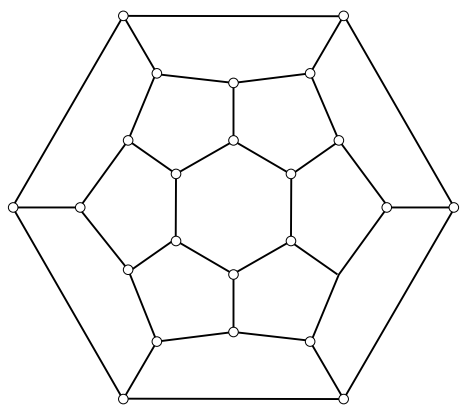

Figure 4: A Löbell 3-polytope $L(6)$

For each $m \geq 5$, the Löbell 3-polytope $L(m)$ has no prismatic 3-or 4-circuits. By Theorem 1.1 almost all compact hyperbolic Coxeter 3 -orbifolds with the combinatorial type of $L(m)$ are weakly orderable.

\subsection{An example satisfying only the condition (C1)}

Let $d$ be a fixed integer greater than 3. We consider the compact hyperbolic Coxeter 3-polytope $P$ shown in Figure 5. Here, if an edge is labeled $d$, then its dihedral angle is $\pi / d$. Otherwise, its dihedral angle is $\pi / 2$.

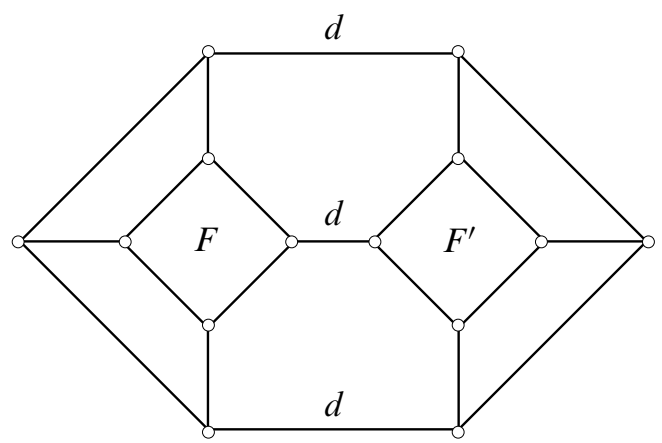

Figure 5: A compact hyperbolic Coxeter 3-polytope

Obviously, $e_{+}(\widehat{P})-3=0$. However $\widehat{P}$ is not weakly orderable, since every facet in $\widehat{P}$ contains four edges of order 2 .

Observe that the cell structure of $P$ has a reflection-type topological symmetry interchanging $F$ and $F^{\prime}$. Hence, the Coxeter 3 -orbifold $\widehat{P}$ arising from $P$ has an order-two isometry fixing an embedded totally geodesic 2-dimensional suborbifold $S$ by the Mostow rigidity. Projective bendings along $S$ provide nontrivial deformations in $\mathbb{D}(\widehat{P})$ by Johnson and Millson [35, Lemma 5.1]. Hence a neighborhood of the 
hyperbolic point in $\mathbb{D}(\widehat{P})$ is not a manifold of dimension 0 while $\mathbb{D}(\widehat{P})$ could still be a manifold; see Choi, Hodgson and Lee [20, Theorem 10] also.

\subsection{An example satisfying only the condition (C2)}

In 1996, Esselmann [27] classified all the compact hyperbolic Coxeter polytopes whose combinatorial types are the products of two simplices of dimension greater than 1 . Let $P$ be the compact hyperbolic Coxeter 4-polytope whose combinatorial type is the product of two triangles and whose Coxeter graph is shown in Figure 6; see Vinberg [48] or Bourbaki [10] for the definition of Coxeter graphs.

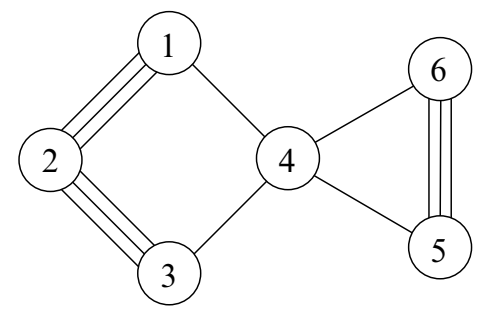

Figure 6: One of Esselmann's polytopes

Since the 4-polytope $P$ has 6 facets and 15 ridges,

$$
\delta_{P}=e-n f+\frac{n(n+1)}{2}=1 \neq 0,
$$

ie $P$ does not satisfy condition $(\mathrm{C} 1)$.

However the Coxeter orbifold $\widehat{P}$ arising from $P$ is weakly orderable, ie $\widehat{P}$ satisfies condition (C2). This can be shown by checking explicitly.

We show that the hyperbolic point in $\mathbb{D}(\widehat{P})$ for the hyperbolic Coxeter orbifold $\widehat{P}$ is singular.

Assume that $\Gamma$ is a projective Coxeter group so that $\Omega_{\Gamma} / \Gamma$ is homeomorphic to $\widehat{P}$, and $A$ is the Cartan matrix of $\Gamma$. We make the Cartan matrix $A$ by a unique diagonal action (see (3-6)) so that

$$
\begin{aligned}
& a_{12}=a_{21}=-2 \cos \left(\frac{\pi}{5}\right), \\
& a_{23}=a_{32}=-2 \cos \left(\frac{\pi}{5}\right), \\
& a_{34}=a_{43}=-2 \cos \left(\frac{\pi}{3}\right), \\
& a_{45}=a_{54}=-2 \cos \left(\frac{\pi}{3}\right), \\
& a_{56}=a_{65}=-2 \cos \left(\frac{\pi}{5}\right) .
\end{aligned}
$$


Define $x=-a_{14}$ and $y=-a_{46}$. The Cartan matrix $A=\left(a_{i j}\right)$ of $\Gamma$ is

$$
A=\left[\begin{array}{ccrrrc}
2 & -\frac{1+\sqrt{5}}{2} & 0 & -x & 0 & 0 \\
-\frac{1+\sqrt{5}}{2} & 2 & -\frac{1+\sqrt{5}}{2} & 0 & 0 & 0 \\
0 & -\frac{1+\sqrt{5}}{2} & 2 & -1 & 0 & 0 \\
-x^{-1} & 0 & -1 & 2 & -1 & -y \\
0 & 0 & 0 & -1 & 2 & -\frac{1+\sqrt{5}}{2} \\
0 & 0 & 0 & -y^{-1} & -\frac{1+\sqrt{5}}{2} & 2
\end{array}\right] .
$$

Moreover, $\operatorname{rank} A=5$ if and only if $\operatorname{det}(A)=0$. By simple calculation, we obtain

$$
\operatorname{det}(A)=\frac{1}{2 x y}\left(8 x-(5+\sqrt{5}) y-(6-2 \sqrt{5}) x y-(5+\sqrt{5}) x^{2} y+8 x y^{2}\right)=0 .
$$

Note that $x$ and $y$ are positive. By Corollary 3.1, the deformation space $\mathbb{D}(\hat{P})$ is homeomorphic to the solution space

$\mathcal{S}=\left\{(x, y) \in \mathbb{R}_{+}^{2} \mid f(x, y):=8 x-(5+\sqrt{5}) y-(6-2 \sqrt{5}) x y-(5+\sqrt{5}) x^{2} y+8 x y^{2}=0\right\}$, pictured in Figure 7.

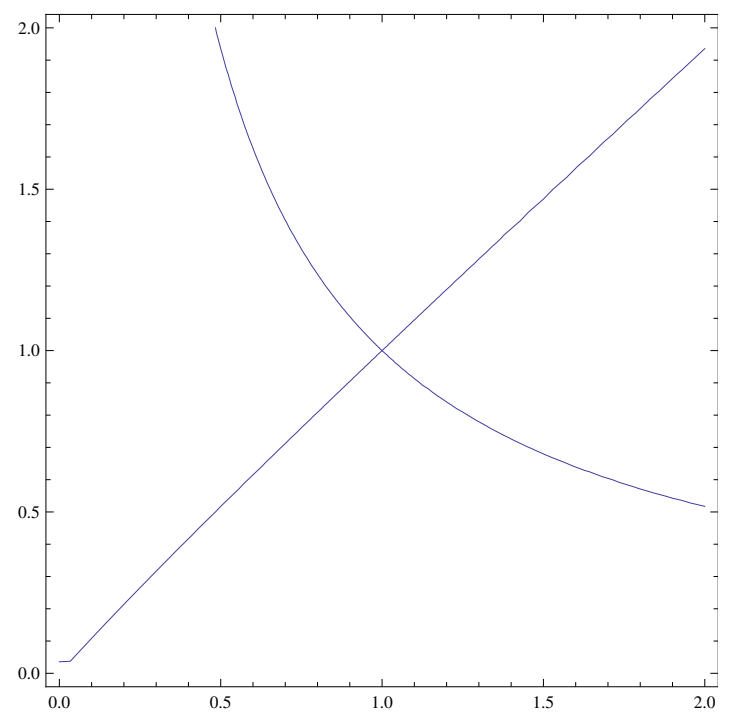

Figure 7: The equation $8 x-(5+\sqrt{5}) y-(6-2 \sqrt{5}) x y-(5+\sqrt{5}) x^{2} y+$ $8 x y^{2}=0$

By Vinberg [47, Proposition 24], $(1,1) \in \mathcal{S}$ corresponds to the unique hyperbolic point in $\mathbb{D}(\widehat{P})$, and hence any neighborhood of the hyperbolic point of $\mathbb{D}(\widehat{P})$ is singular. (The polynomial $f(x, y)$ is irreducible.) 


\section{References}

[1] S Ballas, Deformations of noncompact, projective manifolds arXiv:1210.8419

[2] D Barnette, A proof of the lower bound conjecture for convex polytopes, Pacific J. Math. 46 (1973) 349-354 MR0328773

[3] Y Benoist, Automorphismes des cônes convexes, Invent. Math. 141 (2000) 149-193 MR1767272

[4] Y Benoist, Convexes divisibles, C. R. Acad. Sci. Paris Sér. I Math. 332 (2001) 387-390 MR1826621

[5] Y Benoist, Convexes divisibles, III, Ann. Sci. École Norm. Sup. 38 (2005) 793-832 MR2195260

[6] Y Benoist, Convexes hyperboliques et quasiisométries, Geom. Dedicata 122 (2006) 109-134 MR2295544

[7] Y Benoist, A survey on divisible convex sets, from: "Geometry, analysis and topology of discrete groups", (L Ji, K Liu, L Yang, S-T Yau, editors), Adv. Lect. Math. (ALM) 6, International Press, Somerville, MA (2008) 1-18 MR2464391

[8] Y Benoist, Five lectures on lattices in semisimple Lie groups, from: "Géométries à courbure négative ou nulle, groupes discrets et rigidités”, (L Bessières, A Parreau, B Rémy, editors), Sémin. Congr. 18, Soc. Math. France, Paris (2009) 117-176 MR2655311

[9] J-P Benzécri, Sur les variétés localement affines et localement projectives, Bull. Soc. Math. France 88 (1960) 229-332 MR0124005

[10] N Bourbaki, Lie groups and Lie algebras, Chapters 4-6, Elements of Math., Springer, Berlin (2002) MR1890629

[11] A Brøndsted, An introduction to convex polytopes, Graduate Texts in Math. 90, Springer, New York (1983) MR683612

[12] R Charney, M Davis, When is a Coxeter system determined by its Coxeter group?, J. London Math. Soc. 61 (2000) 441-461 MR1760693

[13] S Choi, Convex decompositions of real projective surfaces, $I$ : $\pi$-annuli and convexity, J. Differential Geom. 40 (1994) 165-208 MR1285533

[14] S Choi, Convex decompositions of real projective surfaces, II: Admissible decompositions, J. Differential Geom. 40 (1994) 239-283 MR1293655

[15] S Choi, The convex and concave decomposition of manifolds with real projective structures, Mém. Soc. Math. Fr. 78, Soc. Math. France, Paris (1999) MR1779499

[16] S Choi, Geometric structures on orbifolds and holonomy representations, Geom. Dedicata 104 (2004) 161-199 MR2043960

[17] S Choi, The deformation spaces of projective structures on 3-dimensional Coxeter orbifolds, Geom. Dedicata 119 (2006) 69-90 MR2247648 
[18] S Choi, Geometric structures on 2-orbifolds: Exploration of discrete symmetry, Math. Soc. Japan Memoirs 27, Math. Soc. Japan, Tokyo (2012) MR2962023

[19] S Choi, W M Goldman, The classification of real projective structures on compact surfaces, Bull. Amer. Math. Soc. 34 (1997) 161-171 MR1414974

[20] S Choi, C D Hodgson, G-S Lee, Projective deformations of hyperbolic Coxeter 3orbifolds, Geom. Dedicata 159 (2012) 125-167 MR2944525

[21] D Cooper, K Delp, The marked length spectrum of a projective manifold or orbifold, Proc. Amer. Math. Soc. 138 (2010) 3361-3376 MR2653965

[22] D Cooper, D D Long, M B Thistlethwaite, Computing varieties of representations of hyperbolic 3-manifolds into SL(4, R), Experiment. Math. 15 (2006) 291-305 MR2264468

[23] D Cooper, D D Long, M B Thistlethwaite, Flexing closed hyperbolic manifolds, Geom. Topol. 11 (2007) 2413-2440 MR2372851

[24] M W Davis, The geometry and topology of Coxeter groups, London Math. Soc. Monographs 32, Princeton Univ. Press (2008) MR2360474

[25] M W Davis, Lectures on orbifolds and reflection groups, from: "Transformation groups and moduli spaces of curves", (L Ji, S-T Yau, editors), Adv. Lect. Math. (ALM) 16, International Press, Somerville, MA (2011) 63-93 MR2883685

[26] M W Davis, When are two Coxeter orbifolds diffeomorphic?, Michigan Math. J. 63 (2014) 401-421 MR3215556

[27] F Esselmann, The classification of compact hyperbolic Coxeter $d$-polytopes with $d+2$ facets, Comment. Math. Helv. 71 (1996) 229-242 MR1396674

[28] J-L Fouquet, H Thuillier, On removable edges in 3-connected cubic graphs, Discrete Math. 312 (2012) 2652-2659 MR2935416

[29] F R Gantmacher, The theory of matrices, Vol. 1, 2, Chelsea Publ., New York (1959) MR0107649

[30] W M Goldman, The symplectic nature of fundamental groups of surfaces, Adv. in Math. 54 (1984) 200-225 MR762512

[31] W M Goldman, Convex real projective structures on compact surfaces, J. Differential Geom. 31 (1990) 791-845 MR1053346

[32] $\mathbf{R}$ Greene, The deformation theory of discrete reflection groups and projective structures, $\mathrm{PhD}$ thesis, Ohio State University (2013) Available at http:// search.proquest. com/docview/1648378685

[33] B Grünbaum, Convex polytopes, 2nd edition, Graduate Texts in Math. 221, Springer, New York (2003) MR1976856

[34] M Heusener, J Porti, Infinitesimal projective rigidity under Dehn filling, Geom. Topol. 15 (2011) 2017-2071 MR2860986 
[35] D Johnson, J J Millson, Deformation spaces associated to compact hyperbolic manifolds, from: "Discrete groups in geometry and analysis", (R Howe, editor), Progr. Math. 67, Birkhäuser, Boston (1987) 48-106 MR900823

[36] V G Kac, È B Vinberg, Quasihomogeneous cones, Mat. Zametki 1 (1967) 347-354 MR0208470

[37] M Kapovich, Deformations of representations of discrete subgroups of $\mathrm{SO}(3,1)$, Math. Ann. 299 (1994) 341-354 MR1275772

[38] J-L Koszul, Déformations de connexions localement plates, Ann. Inst. Fourier (Grenoble) 18 (1968) 103-114 MR0239529

[39] N H Kuiper, On convex locally-projective spaces, from: "Convegno Internazionale di Geometria Differenziale, Italia, 1953”, Edizioni Cremonese, Rome (1954) 200-213 MR0063115

[40] L Marquis, Espace des modules de certains polyèdres projectifs miroirs, Geom. Dedicata 147 (2010) 47-86 MR2660566

[41] D Qi, On irreducible, infinite, nonaffine Coxeter groups, Fund. Math. 193 (2007) 79-93 MR2284573

[42] MS Raghunathan, Discrete subgroups of Lie groups, Springer, New York (1972) MR0507234

[43] R K W Roeder, J H Hubbard, W D Dunbar, Andreev's theorem on hyperbolic polyhedra, Ann. Inst. Fourier (Grenoble) 57 (2007) 825-882 MR2336832

[44] W P Thurston, The geometry and topology of three-manifolds, Princeton Univ. Math. Dept. lecture notes (1979) Available at http://msri.org/publications/books/ gt $3 \mathrm{~m} /$

[45] W P Thurston, Three-dimensional geometry and topology, Vol. 1, Princeton Math. Series 35, Princeton Univ. Press (1997) MR1435975

[46] W T Tutte, The factorization of linear graphs, J. London Math. Soc. 22 (1947) 107-111 MR0023048

[47] ̀ B Vinberg, Discrete linear groups that are generated by reflections, Izv. Akad. Nauk SSSR Ser. Mat. 35 (1971) 1072-1112 MR0302779

[48] ̇ B Vinberg, Hyperbolic groups of reflections, Uspekhi Mat. Nauk 40 (1985) 29-66, 255 MR783604

[49] A Weil, On discrete subgroups of Lie groups, II, Ann. of Math. 75 (1962) 578-602 MR0137793

[50] A Weil, Remarks on the cohomology of groups, Ann. of Math. 80 (1964) 149-157 MR0169956

[51] M Wiemeler, Exotic torus manifolds and equivariant smooth structures on quasitoric manifolds, Math. Z. 273 (2013) 1063-1084 MR3030690 
Department of Mathematical Sciences, KAIST

Daejeon 305-701, South Korea

Mathematisches Institut, Ruprecht-Karls-Universität Heidelberg

D-69120 Heidelberg, Germany

schoi@math.kaist.ac.kr, lee@mathi.uni-heidelberg.de

http://mathsci.kaist.ac.kr/ schoi/,

http://www.mathi. uni-heidelberg.de/ lee/

Proposed: Walter Neumann

Received: 16 July 2012

Seconded: Benson Farb, Danny Calegari

Revised: 23 July 2014 